\title{
Neuroscientific evidence for pain being a classically conditioned response to trauma- and pain-related cues in humans
}

\author{
Laila K. Franke ${ }^{1}$, Stephan F. Miedl ${ }^{1}$, Sarah K. Danböck ${ }^{1}$, Michael Liedlgruber ${ }^{1}$, Markus \\ Grill $^{1}$, Martin Kronbichler ${ }^{2,3}$, Herta Flor ${ }^{4,5}$, Frank H. Wilhelm ${ }^{1}$
}

${ }^{1}$ Division of Clinical Psychology and Psychopathology, Department of Psychology, ParisLodron-University Salzburg, Salzburg, Austria

${ }^{2}$ Centre for Cognitive Neuroscience, Paris-Lodron-University Salzburg, Salzburg, Austria

${ }^{3}$ Neuroscience Institute, Christian Doppler University Hospital, Paracelsus Medical University Salzburg, Salzburg, Austria

${ }^{4}$ Institute of Cognitive and Clinical Neuroscience, Central Institute of Mental Health, Medical Faculty Mannheim, Heidelberg University, Mannheim, Germany

${ }^{5}$ Department of Psychology, School of Social Sciences, University of Mannheim, Mannheim, Germany

Text pages (including references): 37

Number of figures: 7

Number of tables: 3

*Corresponding author: Laila K. Franke: University of Salzburg, Department of Psychology, Hellbrunner Straße 34, 5020, Salzburg, Austria. E-mail address:

lailakatharina.franke@sbg.ac.at. 


\begin{abstract}
Psychological trauma is typically accompanied by physical pain, and posttraumatic stress disorder (PTSD) often co-occurs with chronic pain. Clinical reports suggest that pain after trauma may be part of a re-experiencing symptomatology. Classical conditioning can underlie visual re-experiencing since intrusions can occur as conditioned responses (CRs) to traumarelated cues. If individuals also experience pain to cues previously paired with, but not anymore inflicting nociceptive stimulation (CSs), conditioning could also explain re-experiencing of pain. Sixty-five participants underwent classical conditioning, where painful electrocutaneous stimulation and aversive film-clips served as unconditioned stimuli (USs) in a 2(pain/no pain) $\times 2$ (aversive/neutral film) design. CSs were neutral pictures depicting contextual details from the films. One day later, participants were re-exposed to CSs during a memory-triggeringtask (MTT). We assessed pain-CRs by self-report and an fMRI-based marker of nociceptive pain, the neurologic pain signature (NPS); and recorded spontaneous daily-life pain-intrusions with an e-diary. During conditioning, pain-signaling CSs elicited more self-reported-pain and NPS-responses than no-pain-signaling CSs. Possibly because the aversive-film masked differences in participants' responses to pain-signaling vs. no-pain-signaling CSs, pain-CRs during acquisition only emerged within the neutral-film condition. When participants were reexposed to CSs during MTT, self-reported-pain-CRs during the neutral-film condition and NPS-CRs during the aversive-film condition persisted. Importantly, participants with stronger pain-CRs showed a greater probability and severity of experiencing spontaneous pain intrusions during daily-life. Our data support that pain can emerge as a CR with emotional and sensory components. Classical conditioning presents a possible mechanism explaining painintrusions, and more broadly, pain experienced without nociceptive input.
\end{abstract}


Keywords: classical conditioning, pain conditioning, intrusive memories, trauma film, posttraumatic stress disorder, chronic pain. 


\section{Introduction}

Chronic pain and posttraumatic stress disorder (PTSD) co-occur at striking frequency $[1,72,77,80]$ but mechanisms explaining this comorbidity are poorly understood[55]. Re-experiencing the traumatic event as recurrent, involuntary, and intrusive distressing memories is a core symptom of PTSD[4]. Interestingly, PTSD patients reexperience the traumatic event not only as distressing visual intrusions (e.g., the perpetrator's face), but often (e.g., 49\% of PTSD patients [44]) also as painful sensations[20,29,70]. Possibly, re-experiencing the traumatic event could thus be a factor common to both PTSD and chronic pain.

One way to understand re-experiencing is through classical conditioning. Following conditioning principles, originally neutral stimuli (conditioned stimuli, CSs) become associated with a biologically relevant unconditioned stimulus (US) through temporospatial proximity. Subsequently, CSs usually elicit similar responses to the US (i.e., conditioned responses; CRs). Previously, we showed that CSs not only elicited affective and physiological fear CRs, but also intrusive memories as CRs[20,23,33,74,86]. Critically, studies investigating intrusions as CRs have exclusively used aversive film-clips as US. As such, perhaps unsurprisingly, CRs mostly remained within the visual domain[23]. The inclusion of painful USs might lead to CRs in the somatosensory domain. Thus, here we tested if pain-signaling CSs elicit pain-CRs, just like aversive-footage-signaling CSs have shown to produce fear and visual intrusions as CRs[23,64,75,85].

Most clinicians endorse the idea that pain can be a CR and believe that there is strong evidence for this idea[47]. Further, it has been theorized that pain can occur as a CR that maintains itself beyond the initial source of nociception has subsided[22,51,56,82]. However, experimental evidence for the idea of pain as a CR is scant. Research has mainly evaluated how conditioning modulates pain perception. Results have supported conditioned 
hyperalgesia, i.e., the phenomenon that, after conditioning, individuals perceive nociceptive stimuli as more painful if preceded by pain-signaling CS[4,30,36,46]. On the other hand, studies have also suggested conditioned allodynia, i.e., that, after conditioning, individuals perceive stimuli as painful at a lower threshold if these stimuli are preceded by pain-signaling CS[45,79]. Although evidence suggests that pain-signaling CSs can either amplify pain or render ambiguous nociceptive stimulation to be perceived as painful more easily, whether such CSs also elicit pain in the absence of any nociceptive stimulation remains an open question.

This study investigated whether pain-signaling CSs elicit pain in the absence of nociceptive stimulation. As we ultimately aimed to understand chronic pain after psychological trauma, we investigated pain conditioning within a negative affective context (vs. a neutral affective context). Therefore, we employed painful-stimulation and aversive film-clips ("trauma-films"[35]) as USs, resulting in a 2 (pain/no-pain) $\times 2$ (aversive/neutral film) within-subject Trauma-Pain-Conditioning Paradigm (TPC). We hypothesized that participants acquire pain-CRs to cues paired with painful stimulation during conditioning and retain these pain-CRs during a Memory-Triggering-Task (MTT) one day later as well as during daily-life. During conditioning and MTT, we captured pain by self-report and a functional-magnetic-resonance-imaging (fMRI)-based marker of nociceptive pain, the neurological pain signature (NPS)[83]. We tested whether pain was stronger to pain-signaling vs. no-pain-signaling CSs, and whether this was modulated by CSs' affective context. During daily-life, we captured pain-CRs as self-reported pain-intrusions. 


\section{Methods}

\subsection{Participants}

We tested 74 healthy women between 18 and 35 years. We only tested women as previous research has suggested that men respond differently to aversive film clips than women[95], and were unable to include a sufficiently-sized group of men due to monetary-time constraints. Exclusion criteria were medication use (except for oral contraceptives), cardiovascular, neuroendocrinological, and pain-related disorders, plaster allergies, reports of current mental disorders, absence of self-reported physical and psychological resilience. Furthermore, participants reporting high (more than 2-3 times a week) consumption of extremely violent media were excluded to reduce the odds of including participants for whom exposure to aversive film scenes would potentially elicit insufficient distress. FMRI specific exclusion criteria were pregnancy, ferromagnetic implants, other non-removable metal objects, claustrophobia, and very high $(>35)$ or too low $(<18)$ body mass index. Over the course of the study, ten participants were excluded from analysis due to movement $>3 \mathrm{~mm}$ in $\mathrm{X}, \mathrm{Y}$, or $\mathrm{Z}$ direction during fMRI sessions $(N=3$ in first session; of these, two were also excluded from the second session), suspected brain anomaly $(N=1)$, drop-out due to aversiveness of trauma-analogue situation $(N=3)$, and technical difficulties $(N=3)$, therefore leaving a final sample of $N=64$ for first-session acquisition analyses, and $N=65$ for second-session MTT analyses. The study was approved by the Ethics Committee of the Paris-Lodron University of Salzburg (GZ 7/2019). All participants provided written informed consent prior to participation and were reimbursed either with course credit or 80 Euro.

Current depression, anxiety, and stress symptomatology were assessed with the long version of the Depression-Anxiety-Stress-Scales (DASS[31], German version[58]); trait anxiety was additionally assessed with the State-Trait Anxiety Inventory (STAI[73], German version[41]). Somatization symptoms were measured with the Screening for Somatoform 
Symptoms 7 (SOMS-7)[67]. To characterize the sample in terms of potential PTSD symptomatology, we used the revised Impact of Event Scale (IES-R[32], German version[48]) applied to the most distressing life event. As detailed in Table 1, DASS, STAIT, SOMS-7, and IES-R were within normal ranges.

\section{[insert Table 1 about here]}

\subsection{Stimuli}

\subsubsection{Unconditioned Stimuli (US)}

\subsubsection{Painful stimulation}

Electrocutaneous stimulation was delivered via a re-usable concentric surface electrode with $7 \mathrm{~mm}$ diameter and a platinum pin WASP electrode (Brainbox Ltd, Specialty Developments, Cardiff, Wales) that was attached to participants' inner side of the left calf and was controlled by a Digitimer DS7A constant current stimulator (Digitimer Ltd, Hertfordshire, England). To produce stable stimulus intensities[57], electrocutaneous stimulation was applied in 7-pulse trains with a duration of $988 \mathrm{~ms}$ each, and an inter-stimulus interval of 400-1300 ms, resulting in a total stimulation time of $12.5 \mathrm{~s}$. The first pulse train always coincided with film onset.

Stimulation intensity was determined individually using an established stepwise calibration procedure[63]. Stimulation started at $0.2 \mathrm{~mA}$ and was incremented stepwise by $0.2 \mathrm{~mA}$ until participants verbally reported their perceptual threshold (sensation just noticeable). Stimulation intensity was then further incremented by $0.2 \mathrm{~mA}$ per trial until participants reached their pain threshold (sensation is painful). Subsequently, stimulation intensity was increased stepwise by $5 \%$ of the individually-determined pain threshold until participants reported their pain tolerance (maximum pain tolerance), where maximum pain tolerance was defined as "the moment shortly before one would want to tear the electrode off 
the calf, similarly to when one wants to drop a coffee cup that is too hot". If participants did not reach their pain tolerance after three of these intensity-increasing trials, the current stimulation intensity was increased by $15 \%$ until pain tolerance was reached.

During TPC, the applied electrical stimulation intensity corresponded to the intensity at $30 \%$ between the individual pain threshold and pain tolerance. On a scale ranging from 0 (no sensation) to 5 (painful) to 10 (maximal bearable), individually calibrated stimulus-intensity had to be rated between 6-7. This calibrated stimulus intensity was used for the TPC. If the rating was too low or too high, stimulus-intensity was adjusted by $0.1 \mathrm{~mA}$ and tested repeatedly until the desired rating was achieved.

\subsubsection{Film-clips}

Four film-clips with 16 s duration were extracted from commercial movies. The two aversive film-clips depicted severe interpersonal violence scenes (sexual assault in a tunnel; physical assault with an oxygen bottle) from the movie "Irreversible"[59]. The two neutral film-clips depicted ordinary human social interactions with no aversive content (beach walk; basketball game) and were extracted from "Coach Carter"[17] and "Mr. Jones"[21]. Aversive and neutral film-clips were previously validated for use in trauma-film studies and were matched in content, valence, and arousal[3].

\subsubsection{Conditioned Stimuli (CSs)}

CSs were four images resembling contextual elements of each film-clip: a basketball (basketball game scene), a pier (beach scene), a tunnel (sexual assault scene), and an oxygen bottle (physical assault scene). Each CS had a duration of 4s and immediately preceded USs.

\subsection{Procedures}

\subsubsection{Screening and questionnaires}


After receiving written information about the study's procedure and an informed consent form, participants filled out an initial online screening form checking for inclusion and exclusion criteria. When meeting inclusion criteria, we scheduled individual phone calls to explain the study procedure in detail to participants and answer potential questions. One week before the first fMRI session, participants filled out further online questionnaires concerning trait (STAI-T) and demographic variables.

\subsection{2. fMRI Session 1: Trauma-Pain-Conditioning (TPC) paradigm}

\subsubsection{Pre-TPC paradigm}

FMRI sessions took place at the Christian-Doppler Clinic in Salzburg. Upon arrival at the clinic, participants answered a set of questionnaires (DASS, SOMS-7) to indicate whether they felt mentally and physically stable for participation and provided written informed consent. Following, participants underwent the pain calibration procedure while seated on the MRI table (see 2.2.1.1. Stimuli - Painful Stimulation). After determining the individual stimulation intensity, we acquired an eight-minute long resting-state fMRI sequence. Amongst others, this resting state was intended to accustom participants to the MRI environment.

\subsubsection{TPC}

Before the acquisition phase, each of the four CSs was presented four times for habituation. After habituation, participants rated the CSs on pain and valence.

After habituation, we instructed participants that pictures [CSs] might be followed by film-clips or electrical stimulation. In total, each CS was presented eight times, with a reinforcement rate of 50\%. Thus, in half of the trials (i.e., 4 trials), each CS was paired with one of the four corresponding USs: pain + aversive film, nopain + aversive film, pain + neutral film, nopain + neutral film. Film and pain USs started together i.e., there was no onset 
asynchrony between both stimuli. The pairing of neutral and aversive film-clips with pain vs. no pain was counterbalanced between participants. After acquisition, participants rated CSs and USs on pain and valence. During habituation and acquisition, stimuli were presented in a pseudorandom order (not more than two consecutive stimuli of the same type), with intertrial-intervals (ITIs) ranging between 12 and 16s. See Fig.1A for an overview of the TPC paradigm.

After the TPC paradigm, we acquired another eight-minute resting-state fMRI sequence (results to be presented elsewhere).

\subsubsection{FMRI session 2, 24h after TPC: Memory-Triggering-Task (MTT)}

\subsubsection{Pre-MTT}

As in the first fMRI session, participants indicated whether they felt mentally and physically stable for participation. We also repeated the pain calibration procedure and, once participants were lying in the scanner, showed the aversive film-clip that on day 1 had not been associated with pain for that individual. Then, we instructed participants just like before the acquisition session, namely that pictures [CSs] might be followed by film-clips or electrical stimulation. These steps were taken to keep US-expectancy high during MTT.

\subsubsection{MTT}

During MTT, participants were re-exposed to the CSs from the TPC paradigm. CSs were organized within two runs (early/late), each including four CS-condition blocks (i.e., $\mathrm{CS}_{\text {pain+aversive, }} \mathrm{CS}_{\text {nopain+aversive, }} \mathrm{CS}_{\text {pain+neutral, }} \mathrm{CS}_{\text {nopain+neutral }}$ in randomized order. In each block, CSs were presented three times for $4 \mathrm{~s}$; the interval between CS presentations ranged between 6 and 18s and consisted of a black screen. In total, each block lasted 52s. Following each block, participants indicated subjective pain experienced during the respective block (see 2.4. 
Measures and Outcomes). After MTT, we acquired a high-resolution structural scan. Fig. 1B displays an overview of the MTT procedure.

\section{[insert Figure 1 about here]}

\subsection{Measures and Outcomes}

\subsubsection{Measuring pain}

Self-report has been the gold standard for assessing the experience of pain[18]. However, self-report by itself is not a perfect measure, as it is subject to limitations in self-perception, metacognition, and high inter- and intra-person variability[13,28,78]. In this vein, additional measures tracking nociception (i.e., the neural process of encoding noxious stimuli[34,50]) such as the NPS have been deemed welcome additional measures to assess pain[66]. Here, we therefore assessed pain not only with self-report, but also with the NPS[84].

The NPS has over $90 \%$ sensitivity and specificity in predicting acute pain relative to other salient states, including anticipated pain, pain recall, threat cues[83], general (pictureinduced) negative emotion[18], and observed pain[39]. Concretely, the NPS predicts pain level based on fMRI activity mostly within noxious stimuli intensity-encoding regions. Relative activity levels within these regions are specified as weights, which can be positive (indicating more predicted pain with greater activity) or negative (indicating less predicted pain with greater activity). Positive weights are found in dorsal anterior cingulate cortex (dACC), insula, secondary somatosensory cortex (S2), thalamus; Negative NPS weights are found in structures frequently deactivated by pain, such as the ventral medial prefrontal cortex (vmPFC) and occipital cortex. Since the current investigation aimed to evaluate NPSresponses to visual stimuli, known to activate structures such as the occipital cortex that yield negative NPS weights[39], the inclusion of negative NPS weights may not accurately reflect nociceptive pain (e.g., participants may show low NPS responses to nociceptive stimulation 
during CSs presentations because the visual stimulation counter-acts the expected pain-driven deactivation in occipital regions). As such, we opted for conducting our main analyses with the previously published positive NPS weight mask ('NPS-pos')[43]. For an overview of analyses performed on a manually-edited NPS mask excluding occipital regions only, see Supplement 2.

\subsubsection{Unconditioned responses (URs)}

To check whether Film and Pain manipulations were successful, participants rated each of

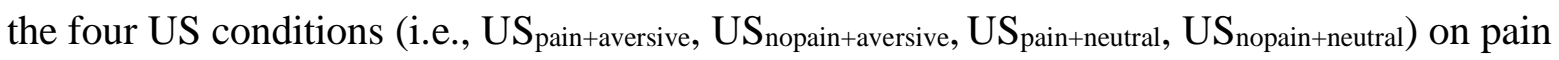
$(0=$ not painful, $10=$ maximal bearable $)$, valence $(0=$ very pleasant, $10=$ very unpleasant $)$, and arousal $(0=$ not arousing at all, $10=$ extremely arousing $)$ at the end of acquisition. Further, we extracted participants' NPS-pos and picture-induced negative affect signature (PINES)[18]) responses, as well as skin conductance responses for each US condition.

\subsubsection{Conditioned responses (CRs)}

\subsubsection{CR acquisition}

This study primarily investigated pain-CRs (self-reported pain, NPS-pos responses), but to better understand which component of pain is conditioned, we secondarily also examined negative-emotion-CRs (self-reported valence, PINES), and SCRs. Self-reported CRs were acquired by asking participants to think back to CS presentations at the end of acquisition. We asked participants to bring back to mind how they felt while viewing the CSs (i.e., $\mathrm{CS}_{\text {pain+aversive, }} \mathrm{CS}$ nopain+aversive, $\left.\mathrm{CS}_{\text {pain+neutral, }} \mathrm{CS}_{\text {nopain+neutral}}\right)$. While showing screenshots of each separate CS, we asked participants to rate how strongly $(0=$ not strong at all, $10=$ maximal bearable) they experienced pain during the respective CSs condition [self-reported pain] and as how pleasant $(0=$ very pleasant, $10=$ very unpleasant $)$ they perceived each CS [valence]. On a neural level, we extracted NPS-pos and PINES responses to the unreinforced CS 
presentations. On a physiological level, we extracted SCRs to each CS presentation during acquisition.

\subsubsection{CR retention (MTT)}

After each MTT block, we asked participants to indicate the percentage of time $(0=0 \%$ of the time, $10=100 \%$ of the time) they experienced painful bodily sensations during the CS block [self-reported pain]. Note that this pain rating differed from the pain rating during TPC (i.e., the analogue 'traumatic event') because we assumed that conditioned pain responses during MTT would rather reflect persistent CRs which could, according to our theory of conditioned intrusions $[22,25,92]$, be conceived of as pain intrusions. Therefore, to capture this potential 'intrusive memory' aspect of persistent pain-CRs, we approximated pain ratings during MTT to previously established measures of conditioned (visual) intrusions[81,92]. Furthermore, within each MTT block, we also extracted NPS-pos and PINES responses, as well as SCRs to the three CS presentations.

\subsubsection{CR retention (daily-life pain intrusions)}

Congruent with the empirically supported assumption that intrusive memories are a form of CR to trauma-related cues[23] we were also curious whether participants would report pain intrusions (next to film intrusions) after conditioning. For this purpose, after TPC we instructed participants to register intrusive memories for seven consecutive days through an e-diary application "PsyDiary" for smartphones[62]. We defined intrusions broadly as spontaneously occurring memories in form of pictures, bodily sensations, sounds, feelings, or thoughts regarding the pain stimulation or film-clips as well as sudden recurring physical sensations, thoughts, or feelings experienced while watching the film clips or experiencing the painful stimulation. We informed participants of the possibility that pain intrusions and film intrusions could mingle and accordingly instructed participants to decide whether their 
memory concerned primarily film clips or painful sensations. Further, we instructed participants to register every intrusion in the e-diary app upon occurrence, i.e., in an eventbased manner. Participants were also asked to report associated distress $(0=$ not at all distressing to $100=$ extremely distressing $)$, and strength of pain sensations $(0=$ not strong at all, $10=$ maximal bearable ) on a visual analogue scale. Finally, participants were also instructed to provide a short content description of the occurring intrusion and potentially occurring triggers in the e-diary app.

To monitor participants' compliance, we sent text-message reminders for a questionnaire at $10 \mathrm{pm}$ each day, where we explicitly assessed whether participants reported all intrusions throughout the day. If participants indicated non-compliance, they were asked to retrospectively estimate the true number and distress of intrusions, and we substituted the event-based intrusion score by the retrospectively estimated intrusion score ( $2 \%$ of data).

\subsection{Apparatus and Physiological Recordings}

Stimulus presentation and behavioral data acquisition were controlled by E-Prime 2.0 (Psychology Software Tools, Inc., Pittsburgh, PA, USA). Skin conductance (SC, in $\mu$ S) was measured using $\mathrm{Ag} / \mathrm{AgCl}$ electrodes filled with isotonic electrode paste[11]; electrodes were placed on the lower palm of the left hand. Recording of SC data was performed with a sampling rate of $1000 \mathrm{~Hz}$ using Polybench 1.22 (TMSi, Twente Medical Systems International, EJ Oldenzaal, Netherlands), a Porti 32-channels-amplifier (TMSi), and an SCamplifier (Becker Meditec, Karlsruhe, Germany). ANSLAB 2.6 was used for SC analysis[9,94].

\subsection{Data analysis}

\subsubsection{FMRI preprocessing and first-level analysis}


FMRI data pre-processing and analysis were performed using SPM12 (Wellcome Department of Cognitive Neurology, London, UK). First, functional images were corrected for geometric distortions by using the FieldMap toolbox, realigned, unwarped, and slice time corrected to the onset of the first slice. Structural images were segmented and normalized to MNI standard stereotactic space. The resulting parameters were then used for normalization of the previously co-registered functional images, which were resampled to isotropic $3 \mathrm{~mm}^{3}$ voxels and smoothed with a $6 \mathrm{~mm}$ full width at half maximum Gaussian kernel. Acquisition and MTT session data were both analyzed in an event-based manner. In the participantspecific first-level model, each event was convolved by a canonical hemodynamic response function. For acquisition analyses, regressors for the first-level model were CS responses during habituation (4s), reinforced and unreinforced CS responses during acquisition (4s), and US responses (16s). For MTT analyses, first-level regressors were CS responses (4s) and regressors of no interest were ratings (10s). In both first-level models, we also added the six rigid-body movement parameters determined from realignment from the respective session as covariates of no interest.

\subsubsection{NPS-pos and PINES analyses}

The expression strength of the NPS-pos and PINES pattern for each of the four US and CS condition was determined by calculating the dot product of the respective vectorized activation images with the NPS-pos and PINES pattern weights. As explained in 2.4.1., we used a mask containing only positive weights for NPS analyses[43].

For US NPS-pos and PINES responses, activation images were individuals' first-level US-response images of each of the four conditions (i.e., US pain+aversive, US $S_{\text {nopain+aversive, }}$ US $_{\text {pain+neutral, }}$ US nopain+neutral), including a total of four trials each. For NPS-pos and PINES responses to CSs during habituation and acquisition, activation images were individuals' first-level CS-response images (i.e., $\mathrm{CS}_{\text {pain+aversive, }} \mathrm{CS}_{\text {nopain+aversive, }} \mathrm{CS}_{\text {pain+neutral, }} \mathrm{CS}_{\text {nopain+neutral}}$ ) 
respectively averaged over the last three CS trials from habituation, two unreinforced CS trials from the first acquisition halve (acq1), and two unreinforced CS trials from the second acquisition halve (acq2).

Similarly, for NPS-pos and PINES responses to CSs during MTT, activation images were individuals' first-level CS-response images, and these were averaged separately over the three CS trials of the early MTT block (MTT1) and over the three CS trials of the late MTT block (MTT2). This returned continuous scalar values for each US and CS condition from habituation, (early and late) acquisition, and early and late MTT.

Higher NPS-pos values indicate higher nociceptive pain[83]; higher PINES values indicate greater picture-induced negative emotion[17].

\subsubsection{Second-level whole brain analyses}

Unreinforced CS responses of each condition were entered into a second-level random effects model. We calculated a full factorial design with Pain (pain vs. no pain) and Film (aversive vs. neutral) as within-participant factors for both acquisition and MTT sessions. For all analyses, the threshold was set to $\mathrm{p}<.05$ corrected for multiple comparisons (based on the false discovery rate (FDR), whole brain level), and a cluster size of $k \geq 5$.

\subsubsection{SCR analyses}

For US analyses, SCRs were quantified by subtracting the mean baseline skin conductance level (SCL, -3 to 0 s relative to the CS onset) from the maximum SCL during each of the four $16 \mathrm{~s}$ film-clips, considering only the first presentation of each film-clip. Similarly, for CR analyses, for each of the four CS conditions we subtracted the mean pre-CS SCL ( -3 to 0 s relative to the CS onset) from the maximum CS SCL (0-5s relative to the CS onset). SCRs were normalized using the natural logarithm of $1+$ SCR. Due to technical problems, data from two participants are missing for SCR analyses. 


\subsubsection{Content and trigger classification of pain intrusions}

For descriptive purposes, we categorized the content of pain intrusions and associated triggers. Based on theoretical expectations, the first author and an experienced graduate student classified pain intrusions based on participants' content description $(1=$ stinging/tingling pain sensations; 2 = other painful sensations; 3 = non-painful bodily sensations; $4=$ combination of painful and non-painful sensations; $5=$ others). Similarly, pain intrusion triggers were classified according to the sensory modality $(1=$ audiovisual; $2=$ painful sensations; $3=$ non-painful bodily sensations; $4=$ thoughts $; 5=$ combination of sensations; $6=$ others). Initial interrater reliabilities were $k=0.68$ for pain intrusion content classification, $k=0.60$ for trigger classification. Disagreements in classifications were discussed until a consensus was reached.

\subsubsection{Statistical analyses}

All statistical analyses were performed in R-Studio[68] in R[65]. For manipulation checks and main analyses, we fitted Bayesian multilevel regression models (BMLMs) $[24,26]$ via the brms package using Stan in R-Studio[13,16]. Rating data (i.e., self-reported pain, valence, arousal) were fitted with ordinal (cumulative) BMLMs[14]. Standardized NPS-pos and PINES responses were fitted with a Gaussian distribution; standardized SCR data were fitted with a skewed normal distribution to account for the left-skewness of the response variable[13].

All models contained repeated measurements over participants (over US-conditions in manipulation checks; and CS-conditions in acquisition/MTT analyses). Thus, to account for the dependency between observations over participants, responses by the same person were modelled with varying intercepts. Furthermore, the predictors Pain and Film were dummy coded (Pain: no pain $=0$, pain $=1$; Film: neutral $=0$, aversive $=1$ ). Since we expected that the effects of Pain and Film on URs and CRs could vary between participants, we added 
varying slopes for Pain and Film in all models to account for this potential variability[6]. For acquisition and MTT analyses, we also added varying slopes for Time in all models. For an overview of fitted models, see Supplement 1.

\subsubsection{Manipulation checks: URs}

To check whether Pain and Film manipulations were successful, we tested whether these experimental factors significantly influenced self-reported pain and NPS-pos responses (pain), self-reported unpleasantness of the film clips and PINES responses (negative emotions), and self-reported arousal and SCRs (arousal) to the four film-clips. For this purpose, we entered Pain and Film, as well as the interaction between Pain $\times$ Film as predictors into BMLMs.

\subsubsection{Main analyses: $C R$ acquisition and retention}

Acquisition. We examined to what extent participants acquired CRs by testing whether the outcome variables (I) self-reported pain, (II) NPS-pos responses, (III) self-reported valence, (IV) PINES responses, and (V) SCRs increased more to CSspain Vs. CSs snopain from habituation to acquisition phases. For this purpose, we added the factors Pain and Time into BMLMs, and tested for Pain $\times$ Time interactions. Time yielded two levels in self-reported pain and valence (habituation $=0, a c q 1+a c q 2=1$ ), and three levels in NPS-pos, PINES, and SCR analyses (habituation $=0, a c q 1=1, a c q 2=2)^{1}$. Since we expected that acquisition of CRs could depend on the affective context induced by our film conditions (avervise vs. neutral), we also tested for Pain $\times$ Time $\times$ Film interactions.

Significant interactions were followed-up by post-hoc BMLMs within each study phase (habituation; acquisition phases) and film conditions (aversive, neutral). To account for

\footnotetext{
${ }^{1}$ This difference in levels is due to participants only rated CSs after habituation and at the end of acq2; imaging data and SCRs were available for both acq1 and acq2.
} 
baseline CSs-valence differences suggested by analyses indicating that participants already rated CSs signaling the aversive-film clip as more unpleasant than CSs signaling the neutralfilm clip during habituation over both Pain conditions, as well as to control for general response tendencies, we corrected acquisition-CRs for habituation-CRs (i.e., acq minus hab). In this way, we used habituation-corrected acquisition CRs in all post-hoc analyses performed on the acquisition phase CRs.

MTT. We examined to what extent participants retained CRs by testing whether the outcome variables (I) self-reported pain, (II) NPS-pos responses, (III) PINES, and (IV) SCRs remained stronger to CSspain vs. CSsnopain during MTT. As the MTT procedure was functionally similar to an extinction procedure, we considered the possibility that participants showed CRs in the first, but not in the second MTT block. As such, we added a Time factor (early $M T T=0$, late $M T T=1)$, and tested for Pain $\times$ Time interactions. Further, we checked whether the retention of CRs depended on the affective context with Pain $\times$ Time $\times$ Film interactions. Significant interactions were followed by post hoc analyses.

\subsubsection{Relationship between self-reported pain and NPS-pos CRs}

We tested whether higher NPS-pos responses were associated with increased selfreported pain at acquisition and MTT by fitting BMLMs[14] where self-reported pain reports were predicted by NPS-pos responses. To test whether the relationship between self-reported pain and NPS-pos responses was modulated by Film and Pain, we added these factors into the model and tested for NPS-pos $\times$ Pain $\times$ Film interactions. Significant interactions were followed up by post-hoc tests. Note that in line with our main analyses, we used habituationcorrected acquisition-CRs in the acquisition analysis. NPS-pos responses were grand-mean centered and standardized before being entered into the BLML. Finally, we added random intercepts for subjects, and random slopes for Pain and Film. 


\subsubsection{Relationship between laboratory-based pain-CRs (self-reported pain, NPS- pos) and daily-life pain intrusions}

As a secondary analysis, we examined whether acquiring and retaining stronger self-reported pain- and NPS-pos-CRs influenced pain intrusions during daily-life. Since persistent PTSD is primarily linked to intrusions perceived as very distressing[49], we aimed to obtain a more clinically relevant variable by weighting pain intrusions by their distress, i.e., operationalizing pain intrusions as "intrusion load" (i.e., daily intrusion number $\times$ average distress; equivalent to the sum of daily intrusive distress). Furthermore, we computed differential CR scores to $\mathrm{CS}_{\text {pain }}$ vs. $\mathrm{CS}_{\text {nopain }}$ within each film condition during acquisition and MTT. Specifically, for the neutral-film condition, we computed difference scores for selfreported pain and NPS-pos responses between CRs to $\mathrm{CS}_{\text {pain }}$ and $\mathrm{CS}_{\text {nopain }}$ within the neutralfilm condition. For the aversive-film condition however, we computed difference scores between $\mathrm{CRs}$ to $\mathrm{CS}_{\text {pain }}$ in the aversive-film condition and $\mathrm{CS}_{\text {nopain }}$ within the neutral-film condition, as the latter condition figured as a more neutral control condition. For acquisition analyses, we used habituation-corrected pain-CR scores over both acquisition trials. For MTT analyses, we also used scores over both early and late MTT.

Given that daily-life intrusions were measured over days, responses by the same person were modelled with varying intercepts. To account for the inflation of zero intrusions in the data, daily-life intrusions were fitted with a hurdle lognormal distribution. With this approach, we fitted data in two parts where (A) estimated the probability of not experiencing (i.e., zero) vs. experiencing (i.e., non-zero) intrusions (hurdle part, modeled as a Bernoulli distribution); and (B) estimated the amount of (i.e., severity of) intrusions $>0$ (lognormal part, modeled with a lognormal distribution[42].

We thus fitted separate models for the predictors (I) self-reported pain and (II) NPS-pos responses during acquisition and MTT. In these models, we further added Day (i.e., 
experimental day on which intrusive memory was registered), as well as interactions between differential pain-CRs and Day to model potential effects of pain-CRs on the persistence/decay of pain intrusions during daily-life[80]. Predictors were centered and standardized before being entered in BMLMs: differential pain-CR scores were centered to their respective means, and Day was centered on the first $24 \mathrm{~h}$ day after TPC, i.e., on the second experimental day. As we expected that the effect of Day on pain intrusions (i.e., the decay of intrusions) could vary between participants, we added a varying slope for the effect of Day.

\subsubsection{BMLM summary statistics}

For a summary of model parameters, we reported regression coefficients and $95 \%$ credible intervals (CIs; i.e., Bayesian confidence intervals). Based upon CIs, we can state that there is a $95 \%$ probability that the respective parameter falls within this interval, given the evidence provided by the data, priors, and model assumptions. Effects were considered significantly different from zero if the estimate's $95 \%$-CIs did not include zero (this would indicate statistical significance on a 5\% level). As priors we used the weak- or non-informative default priors of brms, which have only negligible influence on the obtained results[12,13]. We report Bayesian $R^{2}$ as our measure for effect sizes[25]. All Bayesian models converged according to common algorithms-agnostic[81] and algorithm-specific diagnostics[7]. There were no divergent transitions, Rhat $<1.01$ and $E S S>400$ for all relevant parameters. 


\section{Results}

Results are organized so that a first section (3.1 Manipulation Checks) tests whether Pain and Film (aversive/neutral) manipulations successfully elicited pain, negative emotions, and arousal; a second and a third section (3.2 CR acquisition, 3.3 CR retention) detail to what extent participants acquired and retained pain-CRs (self-reported pain, NPS-pos, pain intrusions), as well as negative emotion-CRs and SCRs within aversive-/neutral-film conditions; a fourth section (3.4 Relationship between self-reported pain and NPS-pos responses) shows the relationship between self-reported pain and NPS responses at acquisition and MTT; and finally a fifth section (3.5 Relationship between laboratory-based pain-CRs (self-reported pain, NPS-pos) and daily-life pain intrusions) tests the relationship between pain-CRs and daily-life pain intrusions.

\subsection{Manipulation Checks: URs}

\subsubsection{Pain}

\subsubsection{Self-report}

Participants reported experiencing more severe pain to the US with pain stimulation (US pain $_{\text {) }}$ than the US with no-pain stimulation (USnopain) $(b=7.20,95 \%-\mathrm{CI}=[5.54,9.17])$. Furthermore, participants also reported experiencing more pain to the aversive-film US (US aversive $_{\text {) than the neutral-film US (US }}$ neutral) $(b=4.60,95 \%-\mathrm{CI}=[3.34,6.13])$. Post-hoc analyses on the significant Pain $\times$ Film interaction $(b=-3.96,95 \%-C I=[-5.38,-2.67])$ within each film condition indicated that the difference between self-reported pain to the US $S_{\text {pain }}$ and $\mathrm{US}_{\text {nopain }}$ was more prominent within the neutral-film $(b=7.87,95 \%-\mathrm{CI}=[1.71,11.83])$ than within the aversive-film condition $(b=2.99,95 \%-\mathrm{CI}=[1.68,5.18])$. See Fig.2A (left) for details. 


\subsubsection{NPS-pos}

In line with the results on self-reported pain, NPS-pos responses suggested greater nociceptive signaling in the $\mathrm{US}_{\text {pain }}$ than in the $\mathrm{US}_{\text {nopain }}$ conditions $(b=0.48,95 \%-\mathrm{CI}=[0.31$, 0.65]). Similarly, nociceptive signaling was greater in the $\mathrm{US}_{\text {aversive }}$ than in the $\mathrm{US}_{\text {neutral }}$ conditions $(b=0.37,95 \%-\mathrm{CI}=[0.23,0.50])$. A non-significant interaction between Pain $\times$ Film suggested that the effect of Pain on NPS-pos responses did not differ between neutral and aversive film-conditions $(b=-0.10,95 \%-\mathrm{CI}=[-0.28,0.08])$. See Fig.2B (right) for details.

\subsubsection{Negative emotions}

\subsubsection{Self-report}

Participants perceived US aversive as more unpleasant than USneutral $(b=6.94,95 \%-C I=[5.66$, 8.36]). Further, participants also perceived the $\mathrm{US}_{\text {pain }}$ as more unpleasant than the US $\mathrm{S}_{\text {nopain }}$ conditions $(b=1.88,95 \%-\mathrm{CI}=[1.17,2.64])$. Post-hoc analyses on the interaction between Pain $\times$ Film $(b=-2.04,95 \%-C I=[-3.05,-1.08])$ suggested that while participants rated the US aversive as equally unpleasant regardless of whether they concomitantly received painful stimulation $(b=-0.11,95 \%-C I=[-1.20,0.57])$, they rated the $\mathrm{US}_{\text {neutral }}$ as more unpleasant when receiving painful stimulation $(b=-2.02,95 \%-C I=[1.19,3.15])$. See Fig. $2 B$ (left) for details.

\subsubsection{PINES}

Participants showed greater PINES responses to US aversive than US $\mathrm{Untral}_{\text {( }}(b=0.66,95 \%-\mathrm{CI}=$ $[0.55,0.77])$. PINES responses did not significantly differ between $\mathrm{US}_{\text {pain }}$ and $\mathrm{US}_{\text {nopain }}(b=$ $0.01,95 \% \mathrm{CI}=[-0.11,0.11])$, and results suggested no significant interactions between Pain and Film $(b=-0.06,95 \% \mathrm{CI}=[-0.22,0.10])$. See Fig. $2 \mathrm{~B}$ (right) for details.

\subsubsection{Arousal}




\subsubsection{Self-report}

Self-reported arousal was higher to the US $\mathrm{Uversive}_{\text {than }}$ to the $\mathrm{US}_{\text {neutral }}(b=4.88,95 \% \mathrm{CI}=$ $[3.80,6.10])$; and higher to $\mathrm{US}_{\text {pain }}$ than to the $\operatorname{US}_{\text {nopain }}(b=1.39,95 \% \mathrm{CI}=[0.70,2.11])$. Furthermore, the significant interaction between Film and Pain $(b=-1.94,95 \% \mathrm{CI}=[-2.78,-$ 0.91]) suggested that whereas participants reported similar arousal to the $\mathrm{US}_{\text {aversive }}$ regardless of whether they concomitantly received painful stimulation $(b=-0.43,95 \% \mathrm{CI}=[-1.18$, 0.28]), participants rated the US neutral as more arousing when this US was paired with painful vs. no-pain stimulation $(b=1.65,95 \% \mathrm{CI}=[0.80,2.85])$. See Fig. $2 \mathrm{C}$ (left) for details.

\subsubsection{SCRS}

Participants showed greater SCRs to the US $\mathrm{Sversive}_{\text {than }}$ to the US neutral $(b=0.59,95 \% \mathrm{CI}=$ $[0.49,0.69])$ and greater SCRs to the $\mathrm{US}_{\text {pain }}$ than to the $\mathrm{US}_{\text {nopain }}(b=0.16,95 \% \mathrm{CI}=[0.06$, 0.26]). The interaction between Pain $\times$ Film was non-significant $(b=-0.09,95 \% \mathrm{CI}=[-0.22$, 0.05]). See Fig. 2C (right) for details.

\section{[insert Figure 2 about here]}

\subsection{CR acquisition}

\subsubsection{Pain}

\subsubsection{Self-report}

Results on self-reported pain to CSs revealed a significant Pain $\times$ Time interaction (see Table 2A.I for regression coefficients and 95\%-CIs). As displayed in Fig. 3A (left) and corroborated by separate post-hoc analyses on self-reported pain to CSs during each study phase (habituation/acquisition), this interaction suggested that participants reported more severe pain to $\mathrm{CS}_{\text {pain }}$ than $\mathrm{CS}_{\text {nopain }}$ during acquisition $(b=2.29,95 \%-\mathrm{CI}=[1.51,3.08])$; during habituation, the difference in self-reported pain responses to $\mathrm{CS}_{\text {pain }}$ vs. $\mathrm{CS}_{\text {nopain }}$ was nonsignificant $(b=1.22,95 \%-\mathrm{CI}=[-0.20,2.66])$. Results further suggested that the factor Film 
(i.e., CSs signaling aversive- vs. neutral-film scenes) modulated the Pain $\times$ Time interaction (see Table 2A.I). Post-hoc analyses on self-reported pain within each study phase revealed that the Pain $\times$ Film interaction was significant during acquisition $(b=-0.89,95 \%-\mathrm{CI}=[-$ $1.67,-0.09])$, but non-significant during habituation $(b=-1.15,95 \%-\mathrm{CI}=[-2.73,0.31])$. Follow-up analyses on the Pain $\times$ Film interaction within each film condition during acquisition suggested that participants reported more pain to $\mathrm{CS}_{\text {pain }}$ than $\mathrm{CS}_{\text {nopain }}$ within the neutral-film condition $(b=1.34,95 \%-C I=[0.61,2.17]$; see upper panel of left Fig. 3A), but also within the aversive-film condition $(b=0.87,95 \%-\mathrm{CI}=[0.13,1.63]$; see lower panel of left Fig. 3A).

\subsubsection{NPS-pos}

Results on NPS-pos responses to CSs revealed significant Pain $\times$ Time interactions (i.e., Pain $\times$ Time_acq1 and Pain $\times$ Time_acq2 interactions, see Table 2A.II for regression coefficients and 95\%-CIs). As displayed in Fig. 3A (right) and corroborated by separate posthoc analyses on NPS-pos responses to CSs within each study timepoint (habituation/acq1+acq2), these interactions suggested that participants showed greater NPSpos responses to $\mathrm{CS}_{\text {pain }}$ than $\mathrm{CS}_{\text {nopain }}$ over both acquisition halves $(b=0.38,95 \%-\mathrm{CI}=[0.16$, $0.61])$, but not during habituation $(b=-0.02,95 \%-C I=[-0.16,0.12])$. Non-significant Pain $\times$ Time (acq1 vs. acq2) interactions suggested that this main-effect of Pain held across both acq1 and acq2 $(b=0.02,95 \%-\mathrm{CI}=[-0.42,0.46])$.

Film modulated the Pain $\times$ Time_acq2 interaction (see Table 2A.II). Post-hoc analyses on NPS-responses revealed a Pain $\times$ Film interaction during acq2 $(b=-0.50,95 \%-\mathrm{CI}=[-0.98,-$ $0.04])$, which was associated with some uncertainty during acq1 $(b=-0.35,95 \%-\mathrm{CI}=[-0.79$, 0.07]). Follow-up post-hoc analyses on the Pain $\times$ Film interaction during acquisition phases suggested that participants showed greater NPS-pos responses to $\mathrm{CS}_{\text {pain }}$ than $\mathrm{CS}_{\text {nopain }}$ within 
the neutral-film condition (acq1: $b=0.36,95 \%-\mathrm{CI}=[0.05,0.66]$, acq $2: b=0.42,95 \%-\mathrm{CI}=$ $[0.05,0.77]$; see upper panel of right Fig. 3A) but not within the aversive-film condition (acq1: $b=0.01,95 \%-\mathrm{CI}=[-0.31,0.32]$, acq $2: b=-0.08,95 \%-\mathrm{CI}=[-0.43,0.28] ;$ see lower panel of right Fig. 3A). During habituation, the Pain $\times$ Film interaction was non-significant $(b$ $=-0.01,95 \%-\mathrm{CI}=[-0.19,0.17])$.

\subsubsection{Negative emotions}

\subsubsection{Self-report}

Analyses suggested a significant interaction between Pain $\times$ Film $\times$ Time (see Table 2B.I). As can be seen from Fig. 3B (left) and suggested by post-hoc tests within each film condition, this interaction concerned participants' ratings to neutral-film CSs: after acquisition, participants rated the neutral-film signaling $\mathrm{CS}_{\text {pain }}$ as more unpleasant than the neutral-film signaling $\mathrm{CS}_{\text {nopain }}(b=1.91,95 \%-\mathrm{CI}=[1.15,2.69])$. During habituation, this difference was non-significant $(b=0.46,95 \%-C I=[-0.29,1.29])$. Within the aversive-film condition, valence ratings did not significantly differ between $\mathrm{CS}_{\text {pain }}$ and $\mathrm{CS}_{\text {nopain }}$ within the aversive-film condition at habituation $(b=-0.61,95 \% \mathrm{CI}=[-0.61,1.29])$ or at acquisition $(b$ $=0.14,95 \%-\mathrm{CI}=[-0.43,0.68])$.

Note however that, possibly because CSs depicted contextual cues from our film-clips (oxygen bottle and tunnel for aversive-film clips/ basketball and pier for neutral-film clips), participants already perceived aversive-film CSs as more unpleasant during habituation in both no-pain $(b=2.71,95 \%-\mathrm{CI}=[1.79,3.96])$ and pain conditions $(b=2.14,95 \%-\mathrm{CI}=$ $[1.27,3.23])$

\subsubsection{PINES}

We observed a significant interaction between Pain $\times$ Film $\times$ Time_acq2 (see Table 2B.II). As suggested by Fig. 3B (right) and post-hoc tests within each film condition and acquisition 
halve, PINES responses were enhanced to $\mathrm{CS}_{\text {pain }}$ vs. CS $\mathrm{CS}_{\text {nopain }}$ within the neutral-film condition in the second half of the acquisition $(b=0.56,95 \%-\mathrm{CI}=[0.22,0.89])$ this difference between $\mathrm{CS}_{\text {pain vs. }} \mathrm{CS}$ nopain within the neutral-film condition was however non-significant within the first acquisition half $(b=-0.04,95 \%-\mathrm{CI}=[-0.36,0.27])$. Further, PINES responses did not significantly differ between $\mathrm{CS}_{\text {pain }}$ and $\mathrm{CS}_{\text {nopain }}$ within the aversive-film condition in the first $(b=-0.26,95 \% \mathrm{CI}=[-0.61,0.10])$ and second half $(b=-0.20,95 \%-\mathrm{CI}=[-0.50,0.11])$ of the acquisition.

\subsubsection{SCRs}

SCRs to CSs were generally greater during acq1, but not significantly modulated by Pain or Film during acquisition, see Table $2 \mathrm{C}$ and Fig. 3C.

$$
\text { [insert Figure } 3 \text { and Table } 2 \text { about here] }
$$

\subsection{CR retention}

\subsubsection{Pain}

\subsubsection{Self-reported pain}

Results on self-reported pain to CSs during MTT revealed that participants reported more pain when re-exposed to $\mathrm{CS}_{\text {pain }}$ than $\mathrm{CS}_{\text {nopain, }}$ and this effect was modulated by Film (see left Fig.4A). As suggested by post-hoc tests within each film condition, participants still reported more pain to the $\mathrm{CS}_{\text {pain }}$ than the $\mathrm{CS}_{\text {nopain }}$ within the neutral-film condition $(b=2.49,95 \%$-CI $=$ $[1.18,3.90])$, such differential pain responses were non-significant within the aversive-film condition $(b=0.11,95 \%-\mathrm{CI}=[-1.01,1.21])$. These effects were similar between early and late MTT blocks, as indicated by non-siginificant interactions between Pain, Time, and Film. See Table 3A.I for regression coefficients and corresponding 95\%-CIs.

\subsubsection{NPS-pos}


We found no significant differences between NPS-pos responses to $\mathrm{CS}_{\mathrm{pain}}$ and $\mathrm{CS}_{\text {nopain. }}$ This held across film conditions and both MTT blocks, as suggested by non-significant Pain $\times$ Film, Pain $\times$ Time, and Pain $\times$ Film $\times$ Time interactions (see Table 3A.II). Yet, considering the pattern of results displayed in Fig.4A.II and the uncertainty associated with the Pain $\times$ Time interaction $(95 \%$-CIs $=-0.85,0.09)$, we examined in an exploratory fashion whether NPS-pos responses differed between $\mathrm{CS}_{\text {pain }}$ and $\mathrm{CS}_{\text {nopain }}$ within the aversive-film condition. NPS-pos responses during early MTT were significantly enhanced to $\mathrm{CS}_{\text {pain }}$ vs $\mathrm{CS}$ nopain during the aversive-film condition $(b=0.38,95 \%-\mathrm{CI}=[0.09,0.66])$. The difference between $\mathrm{CS}_{\text {pain }}$ vs $\mathrm{CS}_{\text {nopain }}$ within the aversive-film condition were non-significant during late $\mathrm{MTT}(b=$ $0.13,95 \%-\mathrm{CI}=[-0.25,0.51])$, or within the neutral-film condition during early $(b=0.14$, $95 \%-\mathrm{CI}=[-0.14,0.44])$ or late $(b=0.13,95 \%-\mathrm{CI}=[-0.92,0.87]) \mathrm{MTT}$.

\subsubsection{Negative emotion (PINES)}

We observed no significant effects of Pain, Film, Time, or any interactions between factors on PINES responses to CSs during MTT. See Table 3B and Fig.4B for details.

\subsubsection{SCRs}

There were no significant effects of Pain, Film, Time, or any interactions between factors on SCRs to CSs during MTT See Table 3C and Fig.4C for details.

[insert Figure 4 and Table 3 about here]

\subsubsection{Daily-life pain intrusions}

Over the seven days following TPC participants reported on average $1.5(S D=2.23)$ spontaneously occurring pain intrusions during daily-life. Participants rated these pain intrusions as moderately distressing $(M=21.22, S D=19.45)$ and strong $(M=24.37, S D=$ 23.01).

Mirroring the pain induced by the electrocutaneous stimulation on the left calf during the TPC paradigm, participants described pain intrusions during daily-life as stinging/tingling 
sensations (48.2\%; e.g., "stinging in my left calf, just like in the MRI, but weaker"), but also as other non-specific painful bodily sensations (9.6\%; e.g., "pain in left calf while playing volleyball"), and non-painful bodily sensations (9.6\%; e.g., "leg cramp"). Some pain intrusions also consisted of a combination of painful/non-painful sensations (13.2\%; "burning sensation on calf and cramping”) or were not further specified (10.3\%).

Some participants (37.2\%) also reported a trigger for their pain intrusions: Triggers included audiovisual input (34.4\%; e.g.: "beach film scene", "seeing the diary application on my smartphone”)), painful bodily sensations (12.5\%; e.g.: “epilating legs"), non-painful bodily sensations (12.5\%; e.g.: "changing position while lying on the couch"), thoughts (25\%; e.g.: "thinking about the study"), a combination between sensations (12.5\%) and other cues $(3.1 \%)$.

\subsection{Relationship between self-reported pain and NPS-pos responses}

\subsubsection{Acquisition}

Analyses of the relationship between self-reported pain and NPS-pos responses during acquisition suggested an interaction between NPS-pos and Pain $(b=0.64,95 \%-\mathrm{CI}=[-0.02$, 1.29]; though note that since confidence intervals contained zero, these results are associated with some uncertainty). Follow-up post-hoc analyses within each pain condition suggested that NPS-pos responses and self-reported pain to CSspain were positively associated $(b=0.60$, $95 \%-\mathrm{CI}=[0.18,1.04])$, indicating that individuals who reported more severe pain at acquisition to CSs sain, also exhibited higher NPS-pos responses to CSspain. Conversely, the relationship between NPS-pos responses and self-reported pain to CSsnopain was still positive, but non-significant $(b=0.16,95 \%-\mathrm{CI}=[-0.05,0.39])$. See Fig. 5 for details. During habituation, the between NPS-pos responses and self-reported pain was also non-significant 
$(b=0.02,95 \%-\mathrm{CI}=[-0.08,0.14])$ and not modulated by the factor Pain $(b=0.01,95 \%-\mathrm{CI}=$ $[-0.15,0.15])$

[insert Figure 5 about here]

\subsubsection{MTT}

At MTT, there was no longer a significant association between NPS-pos responses and selfreported pain $(b=0.36,95 \%-\mathrm{CI}=[-0.27,1.00])$, and this held for both $\mathrm{CS}_{\text {pain }}$ and $\mathrm{CS}_{\text {nopain }}$ as suggested by a non-significant interaction between NPS-pos responses and Pain $(b=0.17$, $95 \%-\mathrm{CI}=[-0.58,0.91])$

\subsection{Relationship between pain-CRs (self-reported pain, NPS-pos) and daily-life pain intrusions}

\subsubsection{Pain-CRs within neutral-film condition}

Self-reported pain. Participants with greater differential self-reported-pain-CRs to $\mathrm{CS}_{\text {pain }}$ vs. $\mathrm{CS}_{\text {nopain }}$ within the neutral-film condition during acquisition and MTT showed greater pain-intrusion severity than participants with lower differential self-reported-pain-CRs (acquisition: $b=0.56,95 \%-\mathrm{CI}=[0.17,0.96]$; MTT: $b=0.43,95 \%-\mathrm{CI}=[0.02,0.85]$, see Figs. 6A.I and 6B.I); this held over days, as suggested by the non-significant interaction between self-reported pain-CRs and Day (acquisition: $b=-0.14,95 \%-\mathrm{CI}=[-0.33,0.04]$; MTT: $b=-0.17,95 \%-\mathrm{CI}=[-0.37,0.03])$. For probability of pain-intrusion absence during daily-life, results suggested no significant effects of the magnitude of differential selfreported-pain-CRs during acquisition or MTT (acquisition: $b=-0.46,95 \%-\mathrm{CI}=[-1.21,0.25]$; MTT: $b=-0.11,95 \%-C I=[-0.83,0.60])$, nor interactions between self-reported-pain-CRs and Day (acquisition: $b=0.16,95 \%-\mathrm{CI}=[-0.09,0.43]$; MTT: $b=0.01,95 \%-\mathrm{CI}=[-0.23$, $0.25]$ ). 
NPS-pos responses. We found no significant effects of differential NPS-pos-CRs to $\mathrm{CS}_{\text {pain }}$ vs. CS $\mathrm{S}_{\text {nopain }}$ within the neutral-film condition during acquisition or MTT (acquisition: $b$ $=0.11,95 \%-\mathrm{CI}=[-0.34,0.57]$; MTT: $b=-0.11,95 \%-\mathrm{CI}=[-0.57,0.34])$ or interactions between NPS-pos-CRs and Day (acquisition: $b=-0.07,95 \%-\mathrm{CI}=[-0.32,0.18]$; MTT: $b=-$ $0.01,95 \%-\mathrm{CI}=[-0.23,0.23])$ on the severity of pain-intrusions during daily-life. However, results suggested that participants who acquired greater differential NPS-pos-CRs at acquisition showed a lower probability of pain-intrusion absence during daily-life $(b=-0.84$, $95 \%-\mathrm{CI}=[-1.68,-0.08])$, which was modulated by Day $(b=0.33,95 \%-\mathrm{CI}=[0.05,0.62])$ : Participants with greater NPS-pos-CRs at acquisition mostly showed a lower probability of pain-intrusion absence during the first days after TPC, which dissipated over the course of days. Conversely, participants with lower NPS-pos-CRs at acquisition showed a constant high probability of pain-intrusion absence over days, see Fig.6A.II for details. Though following the same pattern as during acquisition, during MTT the effects of NPS-pos-CRs ( $b$ $=-0.22,95 \%-\mathrm{CI}=[-0.94,0.54])$ and interaction effect between NPS-pos-CRs and Day $(b=$ $0.19,95 \%-\mathrm{CI}=[-0.07,0.46])$ on pain-intrusion absence during daily-life were nonsignificant.

\section{[insert Figure 6 about here]}

\subsubsection{Pain-CRs within aversive-film condition}

Self-reported pain. Results on the relationship between differential self-reported-painCRs within the aversive film condition and daily-life pain intrusions largely yielded an identical pattern as results within the neutral-film condition but were associated with uncertainty, as zero was included in respective 95\%-CIs. Over acquisition and MTT analyses, no significant effects of self-reported-pain-CRs (acquisition: $b=0.41,95 \%-\mathrm{CI}=[-$ $0.08,0.88]$; MTT: $b=0.03,95 \%-\mathrm{CI}=[-0.42,0.47])$ or significant interaction effects between self-reported pain-CRs and Day (acquisition: $b=-0.10,95 \%-\mathrm{CI}=[-0.26,0.07]$; MTT: $b=-$ 
$0.01,95 \%-\mathrm{CI}=[-0.42,0.47])$ on pain-intrusion severity during daily-life were found.

Similarly, results suggested no significant main effect of self-reported pain-CRs (acquisition: $b=0.01,95 \%-\mathrm{CI}=[-0.73,0.75] ; \mathrm{MTT}: b=0.26,95 \%-\mathrm{CI}=[-0.45,1.03]))$ or significant interaction effects between self-reported pain-CRs and Day (acquisition: $b=-0.04,95 \%-\mathrm{CI}=$ $[-0.30,0.22]$; MTT: $b=-0.19,95 \%-\mathrm{CI}=[-0.42,0.04])$ on daily-life pain-intrusion absence during daily-life were observed.

NPS-pos responses. For pain-intrusion severity during daily-life, analyses on differential NPS-pos-CRs within the aversive-film condition over acquisition and MTT suggested no significant main effect of NPS-pos-CRs (acquisition: $b=-0.40,95 \%-\mathrm{CI}=[-0.86,0.09]$; MTT: $b=0.15,95 \%-\mathrm{CI}=[-0.44,0.75])$ or significant interaction effects between NPS-posCRs and Day (Acquisition: $b=0.08,95 \%-\mathrm{CI}=[-0.11,0.27]$; MTT: $b=-0.11,95 \%-\mathrm{CI}=[-$ $0.30,0.09]))$. Importantly though, results on pain-intrusion absence during daily-life suggested that while NPS-pos-CRs during acquisition did not significantly influence subsequent pain-intrusion absence during daily-life $(b=-0.37,95 \%-\mathrm{CI}=[-1.14,0.39])$, during MTT NPS-pos-CRs significantly predicted the probability of pain-intrusion absence $(b=-1.10,95 \%-\mathrm{CI}=[-2.20,-0.15])$, in that participants with stronger differential NPS-posCRs to $\mathrm{CS}_{\text {pain }}$ during the aversive-film condition at MTT showed a lower probability of painintrusion absence during daily-life (see Fig. 6B.II). These effects held over Days (acquisition: $b=0.20,95 \%-\mathrm{CI}=[-0.06,0.46] ; \mathrm{MTT}: b=0.22,95 \%-\mathrm{CI}=[-0.07,0.53])$.

\subsubsection{Summary}

Overall, significant relationships between pain intrusions and pain-CRs primarily emerged within conditions where participants effectively showed significant differential pain responses to $\mathrm{CS}_{\text {pain }}$ vs. $\mathrm{CS}_{\text {nopain }}$ (see sections 3.2.1. and 3.3.1). Specifically, participants who acquired and retained stronger differential self-reported-pain-CRs to $\mathrm{CS}_{\text {pain }}$ within a neutral affective film context showed greater pain-intrusion severity during daily-life. When self- 
reported-pain-CRs to $\mathrm{CS}_{\text {pain }}$ were acquired within the aversive-film context, this relationship was non-significant but followed a similar pattern. Moreover, NPS-pos-CRs also predicted pain intrusions during daily-life: Participants who acquired stronger differential NPS-posCRs to the $\mathrm{CS}_{\text {pain }}$ within the neutral-film condition showed a lower probability for painintrusion absence during daily-life. However, during MTT, participants who retained stronger differential NPS-pos-CRs to $\mathrm{CS}_{\text {pain }}$ within the aversive-film, but interestingly not within the neutral-film condition, showed a lower probability for pain-intrusion absence during dailylife.

\subsection{Exploratory whole-brain Analyses}

To allow full appreciation of pain conditioning acquisition and retention, we computed an exploratory whole-brain analysis for the effect of Pain $\left(\mathrm{CS}_{\text {pain }}>\mathrm{CS}_{\text {nopain }}\right.$ and $\left.\mathrm{CS}_{\text {pain }}<\mathrm{CS}_{\text {nopain }}\right)$. During acquisition, over both neutral- and aversive-film conditions the contrast $\mathrm{CS}_{\text {pain }}>$ $\mathrm{CS}_{\text {nopain }}$ revealed no significant activations. The interaction contrast between Pain and Film (see Supplement Table S3) further revealed activations in widespread regions involved in salience and pain anticipation/processing including bilateral middle temporal and occipital gyri, bilateral rolandic operculum, bilateral anterior insula, and right supplementary motor area (SMA) $[7,19,54]$. When examining the pain contrast separately within the aversive-film and neutral-film conditions, it became apparent that the main effect of pain in salience and pain processing regions exclusively originated within the neutral-film condition (Fig. 7B). During early and late MTT, there were no statistically significant activation patterns at FDRcorrected $p<0.05$.

[insert Figure 7 about here] 


\section{Discussion}

This study investigated whether pain can occur as a CR to cues signaling, but not inflicting, pain (CSs). Our data largely supported the hypothesis that participants acquire conditioned pain responses: during acquisition, cues signaling pain (CSspain) elicited more self-reported pain and NPS-pos responses than cues signaling no pain (CSsnopain). This was moderated by affective context, in that conditioned pain responses were weaker (self-reported-pain-CRs) or absent (NPS-pos-CRs) during the aversive-film condition. Further, upon re-exposure to CSs during MTT 24h later, participants retained self-reported-pain-CRs within the neutral-film condition only. Evidence for participants also retaining NPS-pos-CRs during MTT was more uncertain, but nominally suggested that NPS-pos-CRs persisted within the aversive-film condition. Overall, our data indicated that participants displaying stronger self-reported-painand NPS-pos-CRs showed a greater probability and severity of pain intrusions during dailylife compared to participants with weaker self-reported-pain- and NPS-pos-CRs.

The finding that cues associated with pain merely on a temporal and contextual level[51] can elicit perceived (self-reported) and sensory (NPS-pos) pain-CRs extends studies showing that classical conditioning endows CSs with the ability to amplify the perception of pain in response to nociceptive stimuli[36,37], or reduce pain thresholds to ambiguous atpain-threshold stimuli[45,51,79]. Our findings thus provide further experimental support for the postulation of pain occurring as a classical CR[27,47]. From a broader perspective, this complements research that has shown that, after classical conditioning, pain-signaling CSs elicit a wide range of pain-related fears (e.g., fear of movement) that may contribute to the transition from acute to chronic pain[52]. Even though the effective theoretical, empirical, and clinical distinction between pain-related-fear- and pain-CRs needs to be scrutinized in future research, here we demonstrate that pain-signaling CSs cannot only elicit pain-related fears but also 'pain' itself. 
Intriguingly, participants still reported more pain to $\mathrm{CS}_{\text {pain }}$ than $\mathrm{CS}_{\text {nopain }}$ beyond the initial learning phase when re-exposed to CSs $24 \mathrm{~h}$ later. Reflecting acquisition patterns where participants showed reduced differential pain responses to $\mathrm{CS}_{\text {pain }}$ vs. CS Snopain within the aversive-film condition, during MTT self-reported-pain CRs only clearly emerged within the neutral- but not within the aversive-film condition. Our data suggest that pain conditioning may have been less clear within the aversive-film condition because participants not only reported pain to the $\mathrm{CS}_{\text {pain, }}$, but to some extent also to the $\mathrm{CS}_{\text {nopain }}$ (and $\mathrm{US}_{\text {nopain }}$ ). One possible explanation for participants reporting pain to these conditions associated with no-pain stimulation could relate to aversive film-clips depicting situations of severe violence and pain in others. Considering research suggesting that individuals can resonate with another person's pain experience and expressions of pain[40], pain responses to $\mathrm{CS}_{\text {nopain }}$ within the aversivefilm condition could reflect participants' mirroring another person's pain experience and raises the possibility that empathic pain may also be subject to conditioning. Ultimately, we believe that the experience of pain to aversive stimuli reflects the multidimensional nature of pain, and supports the idea that there is no direct one-to-one relationship between nociception and pain, i.e., that pain is not only a sensory, but also an emotional experience that may occur despite no actual tissue damage is causing activation of peripheral nociceptors[61].

For the sensory component of conditioned pain, our results suggest that NPS-pos

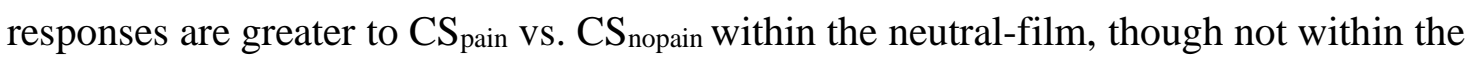
aversive-film condition during acquisition. This agrees with whole-brain-analyses revealing widespread activations in regions involved in salience and pain processing $[7,19,54]$ including bilateral anterior insula, bilateral rolandic operculum, and right supplementary motor area to $\mathrm{CS}_{\text {pain }}>\mathrm{CS}_{\text {nopain }}$ within the neutral-film condition. Noteworthy, this whole-brain-contrast also revealed activations in occipital regions, which could be related to enhanced sensory processing of threat-related cues[2,52,58]. 
Despite previous research suggesting that the NPS-pos does not respond to vicarious pain[39], our data suggest that the aversive-film US overall elicited higher NPS-pos responses than the neutral-film US, and that this was driven, similarly to the self-reported-

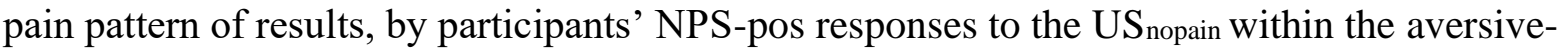
film condition. The aversive-film may thus have somewhat masked the painful stimulation, and thereby also led to reduced differential NPS-pos-CRs to $\mathrm{CS}_{\text {pain }}$ vs. CS $\mathrm{S}_{\text {nopain. Intriguingly, }}$ and although to be considered with care (as based on exploratory post-hoc tests), results suggested that participants show greater NPS-pos-CRs to $\mathrm{CS}_{\text {pain }}$ vs. $\mathrm{CS}_{\text {nopain }}$ within the aversive-film condition during MTT. These results implicate that sensory conditioned pain responses might be more likely to persist when acquired within a distressing/aversive than within a neutral affective context and could thereby reflect the particularly high prevalence of chronic pain in PTSD[1,72,76,77].

Our data further suggest that pain-CRs yield a strong negative emotional component during acquisition. Specifically, CS signaling painful stimulation yet a neutral-film, elicited enhanced conditioned negative emotions as determined by self-report and PINES responses. Interestingly, the temporally more fine-grained PINES responses suggest that conditioned negative emotions to pain only emerged later during acquisition. Given that conditioned NPS-pos responses emerged immediately during the first acquisition halve, acquisition of the sensory component of pain-CRs might be quicker than acquisition of its affective component. Overall, our results support that both sensory and affective components of pain were (at least transiently) conditioned, and thereby suggest that pain-CRs reflect pain as an unpleasant sensory and emotional experience[62].

Altogether, given the current pattern of results, how can we describe the type of pain someone experiences during a pain-CR, and how is this type of pain different from evoked pain? The fact that pain-signaling CSs elicited not only pain perception, but also changes in 
brain activation patterns signalling nociception during acquisition and MTT might suggest that pain-CRs and evoked pain are qualitatively similar. Although a comparison of selfreported pain and NPS-pos responses to the US $\mathrm{Sain}_{\text {and }}$ CS pain suggests that pain-CRs may be weaker than pain-URs, we believe that the most striking difference lays in the fact that, as opposed to evoked pain responses, pain-CRs emerged without any nociceptive input.

Importantly, the finding that nociceptive input does not seem to be necessary to elicit a pain experience with a sensory and negative emotional component supports the idea that there is no direct one-to-one relationship between nociception and pain. Tentatively, we might thus describe pain-CRs as instances of nociplastic pain with a strong negative emotional component, i.e., pain that arises from altered nociception despite no clear evidence of actual or threatened tissue damage causing the activation of peripheral nociceptors or evidence for disease or lesion of the somatosensory system causing the pain[34,38].

More broadly and following empirically supported evidence on the conditioning nature of intrusive memories[20,23,85], we postulated that pain-CRs might form the core of pain-intrusions. Our results supported this by suggesting that participants who demonstrated stronger self-reported-pain- and NPS-pos-CRs during acquisition, and in part also during MTT, subsequently showed a greater probability and severity of pain-intrusions during dailylife. Thereby, this study provides evidence for the critical role of conditioning processes in the etiology of pain-intrusions. We further showed that participants described pain sensations during pain-intrusions similar to those experienced during the experimental pain induction, and occasionally reported film- and pain-related reminder cues as triggers. Interestingly, this resonates with clinical observations in PTSD, including for instance reports of a survivor of bombings who reported re-experiencing the pain in his arms he felt during the explosion when reminded of the trauma[69]; a midwife who, after an unsuccessful resuscitation, kept feeling a painful strain in the hand she used for resuscitating when touching her arm (personal 
communication); or torture survivors describing pain arising from trauma memories[76]. Noteworthy, although we have primarily discussed pain-CRs from the point of view of PTSD research[44], we believe that conditioning mechanisms contributing to pain-CRs might explain persistent pain not only in PTSD, but also be a promising mechanism explaining part of the etiology of nociplastic pain in other chronic pain conditions.

\subsection{Future directions and limitations}

This study reflects the intertwined nature of the pain-emotion relationship and raises questions that require further scrutiny. For example, the fact that pain is unpleasant questions to what extent we can conceive of pain and emotion as separate constructs: does pain just comprise an emotional component (e.g., fear), or do pain and emotion have the same underlying mechanisms, overlapping in such a manner that their distinction is superfluous?[28]. Extending this discussion to the construct of pain-related-fear[52]: how are pain-CRs different to pain-related-fear CRs? Fear and pain are both potent signals to adaptive behaviors such as fight-freeze-flight responses and thus share a strong motivational value. Further, fear and pain are both modulated by a myriad of factors (e.g., cognitions, expectations). Conversely, pain-related-fear- and pain-CRs differ mostly on a sensory level, in that pain-CRs elicit nociceptive signaling in the brain, can be consciously experienced as painful, and consequently be sensed by and referred to localizations within the body. Though emotions also seem to have differential somatic referents in the body, a recent perspective on this topic advanced that it might be the pattern of somatosensory referents and their associated neural manifestation that differentiates between painful and emotional experiences[28]. Clearly, the pain-emotion relationship remains poorly understood on an empirical and theoretical level and should be investigated in future research.

Finally, this study is not without limitations. Findings regarding nociceptive-CRs should be appreciated with care until replicated with other measures of nociception. Since we 
examined participants ${ }^{\prime}$ NPS responses to visual stimuli, including structures where the NPS yields negative weights (e.g., occipital cortex) would lead to low NPS scores. Therefore, we primarily focused on positive NPS weights[84]. Although analyses with NPS-responses including only positive weights did not substantially differ from our analyses with NPSresponses including both weights (Supplement 2), we might not have fully captured the brain response to pain. Thus, it might be informative to investigate the NPS to CSs from other than the visual modality, for instance somatosensory cues[19,53,60]. Furthermore, though we aimed for a minimum number of ratings to reduce subjects' burden and avoid interfering with the immediacy of the learning experience, a more reliable test of whether CSs elicited perceived pain would require participants to rate their pain experience immediately following (or during) each CS presentation, before the US is presented. Further, the use of maximal bearable (vs. unbearable) pain as the upper limit of the pain ratings might have focused the attention of the participants more on tolerability than intensity and could have biased the pain ratings. Finally, it would have been informative to have valence ratings and pain magnitude ratings (i.e., strength of pain sensations) next to NPS-pos and PINES responses also during MTT.

\subsection{Conclusion}

This study results suggests that classical conditioning could be a mechanism underlying pain experiences: The fact that participants acquired and, to some extent, also maintained painCRs implies that temporo-contextually associating CSs with nociceptive stimulation can endow these cues to elicit pain in the absence of noxious stimulation. Further, stronger painCRs appear to enhance the probability and severity of experiencing spontaneous pain intrusions during daily-life. Experiencing pain as a CR to cues associated with pain could thus underly the commonly observed pain re-experiencing in PTSD. Ultimately, pain reexperiencing through conditioning processes may perpetuate pain long beyond tissue healing 
and thereby constitute a mechanism explaining the high comorbidity between chronic pain and PTSD. In this case, interventions such as exposure-based therapies targeting extinction of CRs may prove particularly beneficial in pain management[10].

\section{Acknowledgments}

We thank Tor Wager for sharing the NPS pattern weights. We would also like to thank Michael Martini, Johanna Lohse, Julia Lackner, and Natalie Stöckel for their help in recruitment of participants, data collection, and preliminary analyses. LKF was supported by the Doctoral School Paris-Lodron-University Salzburg Cognitive Neuroscience. SKD was supported by the Doctoral College "Imaging the Mind" (FWF; W1233-B; Project Principal Investigator: FHW). HF was supported by a grant of the Deutsche Forschungsgemeinschaft (SFB1158/B03 to Frauke Nees and HF).

All authors declare no conflicts of interest.

\section{References}

[1] Afari N, Ahumada SM, Wright LJ, Mostoufi S, Golnari G, Reis V, Cuneo JG. Psychological trauma and functional somatic syndromes: A systematic review and meta-analysis. Psychosomatic Medicine 2014;76:2-11.

[2] Antov MI, Plog E, Bierwirth P, Keil A, Stockhorst U. Visuocortical tuning to a threatrelated feature persists after extinction and consolidation of conditioned fear. Scientific Reports 2020;10.

[3] Arnaudova I, Hagenaars MA. Lights ... action: Comparison of trauma films for use in the trauma film paradigm. 2017. doi:10.1016/j.brat.2017.02.007.

[4] Association AP. Diagnostic and statistical manual of mental disorders (DSM-5®). 2013.

[5] Atlas LY, Wager TD. How expectations shape pain. Neuroscience Letters 2012;520:140-148.

[6] Barr DJ, Levy R, Scheepers C, Tily HJ. Random effects structure for confirmatory hypothesis testing: Keep it maximal. Journal of Memory and Language 2013;68:255278.

[7] Betancourt M. A Conceptual Introduction to Hamiltonian Monte Carlo. arXiv 2017.

[8] Biggs EE, Timmers I, Meulders A, Vlaeyen JWS, Goebel R, Kaas AL. The neural correlates of pain-related fear: A meta-analysis comparing fear conditioning studies using painful and non-painful stimuli. Neuroscience and Biobehavioral Reviews 2020;119:52-65. doi:10.1016/j.neubiorev.2020.09.016. 
[9] Blechert J, Peyk P, Liedlgruber M, Wilhelm FH. ANSLAB: Integrated multichannel peripheral biosignal processing in psychophysiological science. Behavior Research Methods 2016;48:1528-1545. doi:10.3758/s13428-015-0665-1.

[10] Bosco MA, Gallinati JL, Clark ME. Conceptualizing and treating comorbid chronic pain and PTSD. Pain Research and Treatment 2013;2013.

[11] Boucsein W, Fowles DC, Grimnes S, Ben-Shakhar G, Roth WT, Dawson ME, Filion DL. Publication recommendations for electrodermal measurements. Psychophysiology 2012;49:1017-1034.

[12] Bürkner PC. Advanced Bayesian multilevel modeling with the R package brms. $\mathrm{R}$ Journal 2018;10:395-411.

[13] Bürkner PC. brms: An R package for Bayesian multilevel models using Stan. Journal of Statistical Software 2017;80. doi:10.18637/jss.v080.i01.

[14] Bürkner P-C, Vuorre M. Ordinal Regression Models in Psychology: A Tutorial. Advances in Methods and Practices in Psychological Science 2019;2:77-101. doi:10.1177/2515245918823199.

[15] de C Williams AC, Davies HT, Chadury Y. Simple pain rating scales hide complex idiosyncratic meanings. Pain 2000;85:457-463.

[16] Carpenter B, Gelman A, Hoffman MD, Lee D, Goodrich B, Betancourt M, Brubaker MA, Guo J, Li P, Riddell A. Stan: A probabilistic programming language. Journal of Statistical Software 2017;76.

[17] Carter T. Coach Carter. USA, 2005.

[18] Chang LJ, Gianaros PJ, Manuck SB, Krishnan A, Wager TD. A sensitive and specific neural signature for picture-induced negative affect. PLoS Biology 2015;13:1-28.

[19] Chang LJ, Gianaros PJ, Manuck SB, Krishnan A, Wager TD. A sensitive and specific neural signature for picture-induced negative affect. PLoS Biology 2015;13:1-28.

[20] Davis KD, Flor H, Greely HT, Iannetti GD, MacKey S, Ploner M, Pustilnik A, Tracey I, Treede RD, Wager TD. Brain imaging tests for chronic pain: Medical, legal and ethical issues and recommendations. Nature Reviews Neurology 2017;13:624-638.

[21] Diesch E, Flor H. Alteration in the response properties of primary somatosensory cortex related to differential aversive Pavlovian conditioning. Pain 2007;131:171-180.

[22] Ehlers A, Hackmann A, Steil R, Clohessy S, Wenninger K, Winter H. The nature of intrusive memories after trauma: The warning signal hypothesis. Behaviour Research and Therapy 2002;40:995-1002.

[23] Figgis M. Mr. Jones. USA, 1993.

[24] Flor H, Birbaumer N. Acquisition of chronic pain. Psychophysiological mechanisms. APS Journal 1994;3:119-127.

[25] Franke LK, Rattel JA, Miedl SF, Danböck SK, Bürkner P-C, Wilhelm FH. Intrusive memories as conditioned responses to trauma cues: An empirically supported concept? Behaviour Research and Therapy 2021;143:103848. doi:10.1016/j.brat.2021.103848.

[26] Gelman A, Carlin JB, Stern HS, Dunson DB, Vehtari A, Rubin DB. Bayesian data analysis. 2013.

[27] Gelman A, Goodrich B, Gabry J, Vehtari A. R-squared for Bayesian Regression Models. American Statistician 2019;73:307-309.

[28] Gelman A, Hill J. Data Analysis Using Regression and Multilevel/Hierarchical Models. Cambridge: Cambridge University Press, 2006 doi:10.1017/CBO9780511790942.

[29] Gentry WD, Bernal GAA. Chronic pain. In: Williams R, Gentry WD., editors. Behavioral approaches to medical treatment. Cambridge: Ballinger, 1977. pp. 173182. 
[30] Gilam G, Gross JJ, Wager TD, Keefe FJ, Mackey SC. What Is the Relationship between Pain and Emotion? Bridging Constructs and Communities. Neuron 2020;107:17-21. doi:10.1016/j.neuron.2020.05.024.

[31] Hackmann A, Ehlers A, Speckens A, Clark DM. Characteristics and content of intrusive memories in PTSD and their changes with treatment. Journal of Traumatic Stress 2004;17:231-240.

[32] Harvie DS, Meulders A, Madden VJ, Hillier SL, Peto DK, Brinkworth R, Moseley GL. When touch predicts pain: Predictive tactile cues modulate perceived intensity of painful stimulation independent of expectancy. Scandinavian Journal of Pain 2016;11:11-18.

[33] Henry JD, Crawford JR. The short-form version of the Depression anxiety stress scales (DASS-21): Construct validity and normative data in a large non-clinical sample. British Journal of Clinical Psychology 2005.

[34] Horowitz M, Wilner N, Alvarez W. Impact of event scale: A measure of subjective stress. Psychosomatic Medicine 1979.

[35] De Houwer J. Revisiting classical conditioning as a model for anxiety disorders: A conceptual analysis and brief review. Behaviour Research and Therapy 2020;127:103558.

[36] IASP. IASP: taxonomy. International Association for the Study of Pain 2021. Available: https://www.iasp-pain.org/terminology?navItemNumber=576\#Pain.

[37] James EL, Lau-Zhu A, Clark IA, Visser RM, Hagenaars MA, Holmes EA. The trauma film paradigm as an experimental psychopathology model of psychological trauma: intrusive memories and beyond. Clinical Psychology Review 2016;47:106-142. doi:10.1016/j.cpr.2016.04.010.

[38] Jepma M, Koban L, van Doorn J, Jones M, Wager TD. Behavioural and neural evidence for self-reinforcing expectancy effects on pain. Nature Human Behaviour 2018;2:838-855.

[39] Jepma M, Wager TD. Conceptual Conditioning. Psychological Science 2015;26:17281739.

[40] Kosek E, Cohen M, Baron R, Gebhart GF, Mico JA, Rice ASC, Rief W, Sluka AK. Do we need a third mechanistic descriptor for chronic pain states? Pain 2016;157:13821386.

[41] Krishnan A, Woo CW, Chang LJ, Ruzic L, Gu X, López-Solà M, Jackson PL, Pujo J, Fan J, Wager TD. Somatic and vicarious pain are represented by dissociable multivariate brain patterns. eLife 2016;5:1-42.

[42] Lamm C, Decety J, Singer T. Meta-analytic evidence for common and distinct neural networks associated with directly experienced pain and empathy for pain. NeuroImage 2011;54:2492-2502.

[43] Laux L, Glanzmann P, Schaffner P, Spielberger C. Das State-Trait Angstinventar. Weinheim (GER): Beltz 1981.

[44] Li N, Elashoff DA, Robbins WA, Xun L. A hierarchical zero-inflated log-normal model for skewed responses. Statistical Methods in Medical Research 2011;20:175189.

[45] López-Solà M, Woo CW, Pujol J, Deus J, Harrison BJ, Monfort J, Wager TD. Towards a neurophysiological signature for fibromyalgia. Pain 2017;158:34-47.

[46] Macdonald B, Salomons T v., Meteyard L, Whalley MG. Prevalence of pain flashbacks in post-traumatic stress disorder arising from exposure to multiple traumas or childhood traumatization. Canadian Journal of Pain 2018;2:48-56. doi:10.1080/24740527.2018.1435994. 
[47] Madden VJ, Bellan V, Russek LN, Camfferman D, Vlaeyen JWS, Moseley GL. Pain by Association? Experimental Modulation of Human Pain Thresholds Using Classical Conditioning. Journal of Pain 2016;17:1105-1115.

[48] Madden VJ, Harvie DS, Parker R, Jensen KB, Vlaeyen JWS, Moseley GL, Stanton TR, Read M. Can Pain or Hyperalgesia Be a Classically Conditioned Response in Humans? A Systematic Review and Meta-Analysis! Data Sources Data Analysis and Meta-Analytical Approach Risk of Bias Classical Conditioning of Hyperalgesia ( $\mathrm{N}=$ 10 ) Exploratory Analysis . n.d.:28-30.

[49] Madden VJ, Moseley GL. Do clinicians think that pain can be a classically conditioned response to a non-noxious stimulus? Manual Therapy 2016;22:165-173.

[50] Maercker A, Schützwohl M. Erfassung von psychischen Belastungsfolgen: Die Impact of Event Skala-revidierte Version (IES-R) [Assessment of post-traumatic stress reactions: The Impact of Event Scale-Revised (IES-R)]. Diagnostica 1998;44:130 141.

[51] Marks EH, Franklin AR, Zoellner LA, Marks EH, Franklin AR, Zoellner LA. Can ' t Get it Out of My Mind: A Systematic Review of Predictors of Intrusive Memories of Distressing Events Can ' t Get it Out of My Mind : A Systematic Review of Predictors of Intrusive Memories of Distressing Events. Psychological Bulletin 2018;144:584640.

[52] McGann JP. Associative learning and sensory neuroplasticity: How does it happen and what is it good for? Learning and Memory 2015;22:567-576.

[53] Merskey $\mathrm{H}$ et al. Pain terms: a list with definitions and notes on usage. Recommended by the IASP Subcommittee on Taxonomy. Pain 1979;6:249.

[54] Meulders A. Fear in the context of pain: Lessons learned from 100 years of fear conditioning research. Behaviour Research and Therapy 2020;131:103635.

[55] Meulders A. Fear in the context of pain: Lessons learned from 100 years of fear conditioning research. Behaviour Research and Therapy 2020;131:103635. doi:10.1016/j.brat.2020.103635.

[56] Meulders A, Vansteenwegen D, Vlaeyen JWS. The acquisition of fear of movementrelated pain and associative learning: A novel pain-relevant human fear conditioning paradigm. Pain 2011;152:2460-2469.

[57] Michelle Welman FHS, Smit AE, Jongen JLM, Tibboel D, van der Geest JN, Holstege JC. Pain Experience is Somatotopically Organized and Overlaps with Pain Anticipation in the Human Cerebellum. Cerebellum 2018;17:447-460.

[58] Moratti S, Keil A. Not what you expect: Experience but not expectancy predicts conditioned responses in human visual and supplementary cortex. Cerebral Cortex 2009;19:2803-2809.

[59] Morgan L, Aldington D. Comorbid chronic pain and post-traumatic stress disorder in UK veterans: a lot of theory but not enough evidence. British Journal of Pain 2020;14:256-262.

[60] Moseley GL, Vlaeyen JWS. Beyond nociception: The imprecision hypothesis of chronic pain. Pain 2015;156:35-38.

[61] Mouraux A, Marot E, Legrain V. Short trains of intra-epidermal electrical stimulation to elicit reliable behavioral and electrophysiological responses to the selective activation of nociceptors in humans. Neuroscience Letters 2014;561:69-73.

[62] MultiMediaTechnology. smarthealthcheck. 2013. Available: https://www.smarthealth.at/project/psydiary-2/. Accessed 28 Jun 2021.

[63] Nilges P, Essau C. Die Depressions-Angst-Stress-Skalen: Der DASS - ein Screeningverfahren nicht nur für Schmerzpatienten. Schmerz 2015;10:649-657.

[64] Noé G. Irreversible. France, 2002. 
[65] De Peuter S, Van Diest I, Vansteenwegen D, Van Den Bergh O, Vlaeyen JWS. Understanding fear of pain in chronic pain: Interoceptive fear conditioning as a novel approach. European Journal of Pain 2011;15:889-894.

[66] Raja SN, Carr DB, Cohen M, Finnerup NB, Flor H, Gibson S, Keefe FJ, Mogil JS, Ringkamp M, Sluka KA, Song X-J, Stevens B, Sullivan MD, Tutelman PR, Ushida T, Vader K. The revised International Association for the Study of Pain definition of pain: concepts, challenges, and compromises. PAIN 2020;161.

[67] Raja SN, Carr DB, Cohen M, Finnerup NB, Flor H, Gibson S, Keefe FJ, Mogil JS, Ringkamp M, Sluka KA, Song X-J, Stevens B, Sullivan MD, Tutelman PR, Ushida T, Vader K. The revised International Association for the Study of Pain definition of pain: concepts, challenges, and compromises. PAIN 2020;161.

[68] Rance M, Ruttorf M, Nees F, Schad LR, Flor H. Neurofeedback of the difference in activation of the anterior cingulate cortex and posterior insular cortex: two functionally connected areas in the processing of pain. Frontiers in Behavioral Neuroscience 2014;8:357. doi:10.3389/fnbeh.2014.00357.

[69] Rattel JA, Wegerer M, Miedl SF, Blechert J, Grünberger LM, Craske MG, Wilhelm FH. Peritraumatic unconditioned and conditioned responding explains sex differences in intrusions after analogue trauma. Behaviour Research and Therapy 2019;116:19-29. doi:10.1016/j.brat.2019.01.009.

[70] RCore Team. R: A Language and Environment for Statistical Computing. Foundation for Statistical Computing 2019. Available: http://www.r-project.org/index.html.

[71] Reddan MC, Wager TD. Modeling Pain Using fMRI: From Regions to Biomarkers. Neuroscience Bulletin 2018;34:208-215. doi:10.1007/s12264-017-0150-1.

[72] Rief W, Hiller W. A New Approach to the Assessment of the Treatment Effects of Somatoform Disorders. Psychosomatics 2003;44:492-498.

[73] Roy-Byrne P, Smith WR, Goldberg J, Afari N, Buchwald D. Post-traumatic stress disorder among patients with chronic pain and chronic fatigue. Psychological medicine 2004;34:363-8. Available: http://www.ncbi.nlm.nih.gov/pubmed/14982142. Accessed 2 Aug 2018.

[74] RStudio Team. RStudio: Integrated development for R [Computer software]. 2020. doi:http://www.rstudio.com/.

[75] Scioli-Salter ER, Forman DE, Otis JD, Gregor K, Valovski I, Rasmusson AM. The shared neuroanatomy and neurobiology of comorbid chronic pain and PTSD: Therapeutic implications. Clinical Journal of Pain 2015;31:363-374.

[76] Sharp TJ, Harvey AG. Chronic pain and posttraumatic stress disorder: Mutual maintenance? Clinical Psychology Review 2001;21:857-877.

[77] Siqveland J, Hussain A, Lindstrøm JC, Ruud T, Hauff E. Prevalence of posttraumatic stress disorder in persons with chronic pain: A meta-analysis. Frontiers in Psychiatry $2017 ; 8$.

[78] Siqveland J, Ruud T, Hauff E. Post-traumatic stress disorder moderates the relationship between trauma exposure and chronic pain. European Journal of Psychotraumatology 2017;8:1375337. doi:10.1080/20008198.2017.1375337.

[79] Spielberger CD, Gorsuch RL, Lushene RE. Manual for the State-Trait Anxiety Inventory. 1970.

[80] Steil R, Ehlers A. Dysfunctional meaning of posttraumatic intrusions in chronic PTSD. Behaviour research and therapy 2000;38:537-58. doi:10.1016/s0005-7967(99)000698 .

[81] Streb M, Conway MA, Michael T. Conditioned responses to trauma reminders: How durable are they over time and does memory integration reduce them? Journal of 
Behavior Therapy and Experimental Psychiatry 2017;57:88-95. doi:10.1016/j.jbtep.2017.04.005.

[82] Streb M, Conway MA, Michael T. Conditioned responses to trauma reminders: How durable are they over time and does memory integration reduce them? Journal of Behavior Therapy and Experimental Psychiatry 2017;57:88-95.

[83] Taylor B, Carswell K, Williams AC de C. The Interaction of Persistent Pain and PostTraumatic Re-Experiencing: A Qualitative Study in Torture Survivors. Journal of Pain and Symptom Management 2013;46:546-555.

[84] Toblin RL, Mack KA, Perveen G, Paulozzi LJ. A population-based survey of chronic pain and its treatment with prescription drugs. Pain 2011;152:1249-1255.

[85] Tracey I, Woolf CJ, Andrews NA. Composite Pain Biomarker Signatures for Objective Assessment and Effective Treatment. Neuron 2019;101:783-800.

[86] Traxler J, Madden VJ, Moseley GL, Vlaeyen JWS. Modulating pain thresholds through classical conditioning. PeerJ 2019;2019:1-20.

[87] Tsur N, Defrin R, Lahav Y, Solomon Z. The traumatized body: Long-term PTSD and its implications for the orientation towards bodily signals. Psychiatry Research 2018;261:281-289.

[88] Vehtari A, Gelman A, Simpson D, Carpenter B, Bürkner P-C. Rank-normalization, folding, and localization: An improved Rb for assessing convergence of MCMC. Bayesian Analysis 2020:1-28.

[89] Vlaeyen JWS, Crombez G. Behavioral Conceptualization and Treatment of Chronic Pain. Annual Review of Clinical Psychology 2020;16:187-212.

[90] Wager TD, Atlas LY, Lindquist MA, Roy M, Woo C-W, Kross E. An fMRI-Based Neurologic Signature of Physical Pain. New England Journal of Medicine 2013;368:1388-1397.

[91] Wager TD, Atlas LY, Lindquist MA, Roy M, Woo C-W, Kross E. An fMRI-Based Neurologic Signature of Physical Pain. New England Journal of Medicine 2013;368:1388-1397.

[92] Wegerer M, Blechert J, Kerschbaum H, Wilhelm FH. Relationship between fear conditionability and aversive memories: evidence from a novel conditioned-intrusion paradigm. PloS one 2013;8:e79025. doi:10.1371/journal.pone.0079025.

[93] Wegerer M, Blechert J, Wilhelm FH. Emotionales Lernen: Ein naturalistisches experimentelles Paradigma zur Untersuchung von Angsterwerb und Extinktion mittels aversiver Filme. Zeitschrift fur Psychiatrie, Psychologie und Psychotherapie 2013;61:93-103.

[94] Wilhelm FH, Peyk P. ANSLAB: Autonomic nervous system laboratory (Version 4.0). 2005. Available: www.sprweb.org.

[95] Wilhelm FH, Rattel JA, Wegerer M, Liedlgruber M, Schweighofer S, Kreibig SD, Kolodyazhniy V, Blechert J. Attend or defend? Sex differences in behavioral, autonomic, and respiratory response patterns to emotion-eliciting films. Biological Psychology 2017;130:30-40. 


\section{Figure Legends}

Figure 1. Experimental design. On day 1, participants underwent the Trauma Pain Conditioning (TPC) paradigm, a classical conditioning procedure with a 2 (aversivefilm/neutral-film) x 2 (pain/no-pain stimulation) within-subject design. The TPC consisted of a habituation phase, where each CS was presented four times without US; and an acquisition phase, where each CS was presented eight times with and reinforcement rate of 50\% (Panel A). Twenty-four hours later, participants returned to the lab and were re-exposed to CSs from TPC during a Memory-Triggering-Task (MTT) during two blocks with three CS presentations (Panel B). After TPC, participants also reported pain-intrusions during daily-life in a diary using a smartphone application (Panel C). Abbreviations: CS = conditioned stimulus; US = unconditioned stimulus; $\mathrm{s}=$ seconds; ITI = intertrial interval; $\mathrm{A}=$ aversive film-clip; $\mathrm{N}=$ neutral film-clip; $\mathrm{P}=$ pain stimulation; $\mathrm{nP}=$ no-pain stimulation.

Figure 2. Fitted values of regressions estimating unconditioned responses (URs) to each US condition. Error bars represent 95\%-CIs, asterisks mark statistical significance. Panel A depicts pain-URs: self-reported pain $(0=$ not painful, $10=$ maximal bearable $)$, and NPS-pos responses. Panel $\mathrm{B}$ depicts negative-emotion-URs: valence ratings $(0=$ very pleasant, $10=$ very unpleasant $)$ and PINES responses. Panel C depicts arousal-CRs: self-reported arousal $(0=n o t$ arousing at all, $10=$ very arousing) and SCRs measured in microSiemens $(\mu \mathrm{S})$. Abbreviations: hab $=$ habituation; acq $=$ acquisition with trials averaged over both acquisition halves; acq1 = first acquisition halve; acq2 = second acquisition halve; NPS-pos = neurologic pain signature responses based on positive weights only; PINES = picture-induce negative affect signature; SCRs $=$ skin conductance responses. 
Figure 3. Fitted values of regressions estimating conditioned responses (CRs) to each CS condition during acquisition. Error bars represent 95\%-CIs, asterisks mark statistical significance. Panel A depicts pain-CRs: self-reported pain $(0=$ not painful, $10=$ maximal bearable) left, and NPS-pos responses right. Panel B depicts negative-emotion-CRs: valence ratings $(0=$ very pleasant, $10=$ very unpleasant $)$ left, and PINES responses right. Panel C depicts SCRs measured in microSiemens $(\mu \mathrm{S})$. Abbreviations: hab = habituation; acq = acquisition with trials averaged over both acquisition halves; acq1 = first acquisition halve; acq2 $=$ second acquisition halve; NPS-pos $=$ neurologic pain signature responses based on positive weights only; PINES = picture-induce negative affect signature; SCRs $=$ skin conductance responses.

Figure 4. Fitted values of regressions estimating conditioned responses (CRs) to each CS condition during MTT. Error bars represent 95\%-CIs, asterisks mark statistical significance. Panel A depicts pain-CRs: self-reported pain $(0=$ not painful, $10=$ maximal bearable $)$ left, and NPS-pos responses right. Panel B depicts negative-emotion-CRs: PINES responses. Panel C depicts SCRs measured in microSiemens $(\mu \mathrm{S})$. Abbreviations: MTT = Memory-TriggeringTask; MTT1 = early MTT; MTT2 = late MTT; NPS-pos = neurologic pain signature responses based on positive weights only; PINES = picture-induce negative affect signature; SCRs = skin conductance responses.

Figure 5. Fitted values on analyses predicting the relationship between self-reported pain and NPS-pos responses to CSspain vs. CSsnopain during acquisition. Shaded areas represent 95\% credible intervals. For illustrative purposes and better appreciation, plots depict non-meancentered NPS-pos estimates. Both self-reported pain and NPS-pos responses were corrected 
for habituation scores. Abbreviations: NPS-pos = neurologic pain signature with positive weights only.

Figure 6. Relationship between spontaneous pain intrusions over days and differential (I) selfreported-pain-CRs and (II) NPS-pos-CRs to CS pain vs. CS nopain during acquisition (A) and MTT (B). Lines in plots A.I and B.I depict fitted values of regressions' lognormal part (i.e., estimating the amount of pain-intrusions [pain-intrusion severity]; Lines in panel A.II and B.II depict fitted valued of the model's hurdle part (i.e., estimating the probability of "zero" painintrusions [pain-intrusion absence]). Shaded areas represent 95\% credible intervals. Abbreviations: $\mathrm{CS}=$ conditioned stimulus; $\mathrm{CS} \_\mathrm{NP}=\mathrm{CS}$ paired with neutral-film + painful stimulation; $\mathrm{CS} \_\mathrm{AP}=\mathrm{CS}$ paired with aversive-film + painful stimulation; $\mathrm{CS} \_\mathrm{NnP}=\mathrm{CS}$ paired with neutral film-clip + no-pain stimulation; NPS-pos = neurologic pain signature responses based on positive weights only; acq = acquisition; MTT = Memory-Triggering-Task.

Figure 7. Activation clusters revealed by the whole brain analyses. Panel A = Interaction Pain $\times$ Film during acquisition irrespective of Film (F-test); Panel B $=$ Pain > NoPain within neutralfilm condition at acquisition (t-test). All clusters were extracted at a threshold of FDR-corrected $p<0.05 ; \mathrm{k} \geq 5$. 
Table 1. Sample characteristics.

\begin{tabular}{lccc}
\hline & $M$ & $S D$ & Range in sample \\
Age (years) & 21.94 & 3.10 & {$[18 ; 31]$} \\
DASS - D & 3.51 & 4.08 & {$[0 ; 15]$} \\
DASS - A & 2.59 & 3.37 & {$[0 ; 15]$} \\
DASS - S & 5.74 & 5.66 & {$[0 ; 22]$} \\
STAI-T & 38.22 & 8.54 & {$[21 ; 61]$} \\
SOMS-7 ICD-10 somatization index & 0.92 & 1.35 & {$[0 ; 6]$} \\
IES-R & -3.17 & 1.65 & {$[-4.44 ; 1.59]$} \\
\hline Note. Values indicating non-clinical relevance are < 10 for DASS - D, < 8 for DASS - A, < 15 for \\
DASS - S, <39-40 for STAI-T[40], and <0 for IES-R. Average ICD-10 somatization scores within \\
the general German population are $M=1.1$ (SD = 1.7)[72]. Abbreviations: DASS - D/A/S = \\
Depression-Anxiety-Stress-Scales; STAI-T = State Trait Anxiety Inventory; SOMS-7 = Screening \\
for Somatoform Symptoms (7-item version); IES-R = Impact-of-Events Scale (Revised version). \\
\hline
\end{tabular}




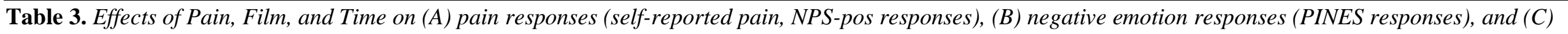
SCRs to CSs during MTT

\begin{tabular}{|c|c|c|c|c|c|}
\hline \multirow{2}{*}{$\begin{array}{l}\text { - Pain } \\
A . I-\text { Self-reported pain }\end{array}$} & & & \multicolumn{3}{|l|}{ B-Negative emotion (PINES) } \\
\hline & $b$ & $95 \%-C I$ & & $b$ & $95 \%-C I$ \\
\hline Pain & 2.55 & {$[1.36,3.80]$} & Pain & 0.09 & {$[-0.25,0.43]$} \\
\hline Film & 2.26 & {$[1.02,3.51]$} & Film & -0.10 & {$[-0.41,0.21]$} \\
\hline Time_MTT2 & -0.70 & {$[-1.63,0.24]$} & Time_MTT2 & -0.17 & {$[-0.50,-0.15]$} \\
\hline Pain $\times$ Film & -2.41 & {$[-3.72,-1.16]$} & Pain $\times$ Film & 0.04 & {$[-0.41,0.21]$} \\
\hline Pain $\times$ Time_MTT2 & 0.28 & {$[-0.92,1.46]$} & Pain $\times$ Time_MTT2 & 0.09 & {$[-0.37,0.53]$} \\
\hline Film $\times$ Time_MTT2 & 0.07 & {$[-1.18,1.31]$} & Film $\times$ Time_MTT2 & 0.29 & {$[0.16,0.73]$} \\
\hline \multirow[t]{2}{*}{ Pain $\times$ Film $\times$ Time_mtt2 } & -0.06 & {$[-1.68,1.57]$} & Pain $\times$ Film $\times$ Time_MTT2 & -0.23 & {$[-0.85,0.42]$} \\
\hline & $R^{2}[95 \%-C I] \quad 0.77$ & {$[0.72,0.81]$} & $R^{2}[95 \%-C I]$ & 0.20 & {$[0.10,0.33]$} \\
\hline
\end{tabular}

\section{A.II - NPS-pos}

Pain

Film

Time_MTT2

Pain $\times$ Film

Pain $\times$ Time_MTT2

Film $\times$ Time_MTT2

Pain $\times$ Film $\times$ Time_MTT2

$\begin{array}{ccc}0.15 & {[-0.16,0.45]} \\ -0.15 & {[-0.45,0.15]} \\ 0.09 & {[-0.21,0.39]} \\ 0.23 & {[-0.18,0.65]} \\ -0.39 & {[-0.85,0.09]} \\ & 0.10 & {[-0.33,0.523]} \\ & 0.14 & {[-0.51,0.81]} \\ R^{2}[95 \%-C I] & 0.23 & {[0.09,0.35]}\end{array}$

\section{$C-S C R s$}

Pain

Film

Time_MTT2

Pain $\times$ Film

Pain $\times$ Time_MTT2

Film $\times$ Time_MTT2

Pain $\times$ Film $\times$ Time_MTT2

$$
R^{2}[95 \%-C I] \quad 0.23 \quad[0.09,0.35]
$$

$\begin{array}{rr}0.12 & {[-0.04,0.29]} \\ 0.07 & {[-0.09,0.24]} \\ 0.01 & {[-0.16,0.19]} \\ -0.13 & {[-0.35,0.09]} \\ -0.05 & {[-0.27,0.16]} \\ -0.01 & {[-0.23,0.21]} \\ 0.08 & {[-0.20,0.37]} \\ R^{2}[95 \%-C I] 0.01 & {[0.01,0.03]}\end{array}$

Notes. Coefficients are considered significantly different from zero if the corresponding 95\% CI does not contain zero and are highlighted in bold. The factor Time was centered on MTT1. For improved readability, we do not display intercepts. Abbreviations: MTT = Memory Triggering Task; MTT1 = early MTT; MTT2 = late MTT; CSs = conditioned stimuli; NPS-pos = neurologic pain signature responses based on positive weights only; PINES = picture-induce negative affect signature. SCRs $=$ skin conductance responses. 
Table 2. Effects of Pain, Film, and Time on (A) pain responses (self-reported pain, NPS-pos responses), (B) negative emotion responses (self-reported valence, PINES responses), and (C) SCRs to CSs during acquisition

\begin{tabular}{|c|c|c|c|c|c|c|c|}
\hline A - Pain & & & & B-Negative emotion & & & \\
\hline A.I - Self-reported pain & & $b$ & $95 \%-C I$ & B.I-Self-reported valence & & $b$ & $95 \%-C I$ \\
\hline Pain & & 0.59 & {$[-0.33,1.53]$} & Pain & & 0.73 & {$[-0.22,1.69]$} \\
\hline Film & & 0.74 & {$[-0.19,1.65]$} & Film & & 3.62 & {$[2.65,4.69]$} \\
\hline Time_acq12 & & -0.39 & {$[-1.45,0.65]$} & Time_acq12 & & -1.09 & {$[-1.84,-0.37]$} \\
\hline Pain $\times$ Film & & -0.51 & {$[-1.76,0.75]$} & Pain $\times$ Film & & -1.37 & {$[-2.49,-0.24]$} \\
\hline Pain $\times$ Time_acq12 & & 3.85 & {$[2.49,5.24]$} & Pain $\times$ Time_acq12 & & 2.65 & {$[-1.53,3.86]$} \\
\hline Film $\times$ Time_acq12 & & 2.60 & {$[1.27,3.92]$} & Film $\times$ Time_acq12 & & 2.09 & {$[0.90,3.29]$} \\
\hline Pain $\times$ Film $\times$ Time_acq12 & & -2.73 & {$[-4.46,-1.01]$} & Pain $\times$ Film $\times$ Time_acq12 & & -2.25 & {$[-3.66,-0.86]$} \\
\hline & $R^{2}[95 \%-C I]$ & 0.58 & {$[0.52,0.63]$} & & $R^{2}[95 \%-C I]$ & 0.73 & {$[0.66,0.79]$} \\
\hline
\end{tabular}

\section{A.II - NPS-pos}

Pain

Film

Time_acq1

Time_acq2

Pain $\times$ Film

Pain $\times$ Time_acq1

Pain $\times$ Time_acq2

Film $\times$ Time_acq 1

Film $\times$ Time_acq2

Pain $\times$ Film $\times$ Time_acq1

Pain $\times$ Film $\times$ Time_acq2

$\begin{array}{lll}-0.02 & {[-0.17,0.13]} & \text { Pain } \\ 0.06 & {[-0.09,0.22]} & \text { Film } \\ -0.10 & {[-0.39,0.19]} & \text { Time_acq1 } \\ -0.12 & {[-0.46,0.21]} & \text { Time_acq2 } \\ -0.01 & {[-0.22,0.20]} & \text { Pain } \times \text { Film } \\ \mathbf{0 . 4 6} & {[\mathbf{0 . 0 9}, \mathbf{0 . 8 2}]} & \text { Pain } \times \text { Time_acq1 } \\ \mathbf{0 . 5 3} & {[\mathbf{0 . 1 3}, \mathbf{0 . 9 5}]} & \text { Pain } \times \text { Time_acq2 } \\ 0.02 & {[-0.41,0.44]} & \text { Film } \times \text { Time_acq1 } \\ 0.16 & {[-0.27,0.59]} & \text { Film } \times \text { Time_acq2 } \\ -0.45 & {[-1.00,0.12]} & \text { Pain } \times \text { Film } \times \text { Time_acq1 } \\ \mathbf{- 0 . 6 4} & {[-\mathbf{- 1 . 2 0}, \mathbf{- 0 . 1 0}]} & \text { Pain } \times \text { Film } \times \text { Time_acq2 }\end{array}$

\section{B.II - PINES}

\begin{tabular}{|c|c|c|}
\hline Pain & 0.10 & {$[-0.15,0.35]$} \\
\hline Film & 0.01 & {$[-0.22,0.33]$} \\
\hline Time_acq1 & -0.24 & {$[-0.52,0.03]$} \\
\hline Time_acq2 & -0.50 & {$[-0.80,-0.19]$} \\
\hline Pain $\times$ Film & -0.09 & {$[-0.43,0.25]$} \\
\hline Pain $\times$ Time_acq1 & -0.05 & {$[-0.41,0.31]$} \\
\hline Pain $\times$ Time_acq2 & 0.65 & {$[0.29,1.01]$} \\
\hline Film $\times$ Time_acq1 & 0.18 & {$[-0.20,0.57]$} \\
\hline Film $\times$ Time_acq2 & 0.57 & {$[0.20,0.92]$} \\
\hline Pain $\times$ Film $\times$ Time_acq1 & -0.25 & {$[-0.81,0.32]$} \\
\hline \multirow[t]{2}{*}{ Pain $\times$ Film $\times$ Time_acq2 } & -0.88 & {$[-1.32,-0.44]$} \\
\hline & 0.61 & {$[0.49,0.71]$} \\
\hline
\end{tabular}




\section{Arousal (SCRs)}

Pain

Film

Time_acq1

Time_acq2

Pain $\times$ Film

Pain $\times$ Time_acq1

Pain $\times$ Time_acq2

Film $\times$ Time_acq 1

Film $\times$ Time_acq2

Pain $\times$ Film $\times$ Time_acq1

Pain $\times$ Film $\times$ Time_acq2

\begin{tabular}{cc}
$b$ & \multicolumn{1}{c}{$95 \%-C I$} \\
0.05 & {$[-0.09,0.21]$} \\
0.06 & {$[-0.07,0.20]$} \\
$\mathbf{0 . 2 6}$ & {$[\mathbf{0 . 0 8}, \mathbf{0 . 4 5}]$} \\
0.03 & {$[-0.14,0.21]$} \\
-0.10 & {$[-0.30,0.08]$} \\
0.19 & {$[-0.05,0.43]$} \\
0.17 & {$[-0.05,0.38]$} \\
0.06 & {$[-0.19,0.30]$} \\
0.07 & {$[-0.14,0.27]$} \\
-0.18 & {$[-0.51,0.15]$} \\
-0.08 & {$[-0.36,0.21]$} \\
0.49 & {$[0.34,0.61]$}
\end{tabular}

Notes. Coefficients are considered significantly different from zero if the corresponding 95\% CI does not contain zero and are highlighted in bold. The factor Time yields two levels for analyses on self-reported pain (habituation, full acquisition [rating was at end of acquisition]), and three levels for NPS-pos, PINES, and SCR analyses (habituation, acq1 $=$ first acquisition halve, acq $2=$ second acquisition halve). Time was centered on habituation scores across analyses. For improved readability, we do not display intercepts. Abbreviations: acq = acquisition; CSs = conditioned stimuli; NPS-pos $=$ neurologic pain signature responses based on positive weights only; PINES = picture-induce negative affect signature. SCRs = skin conductance responses. 
A
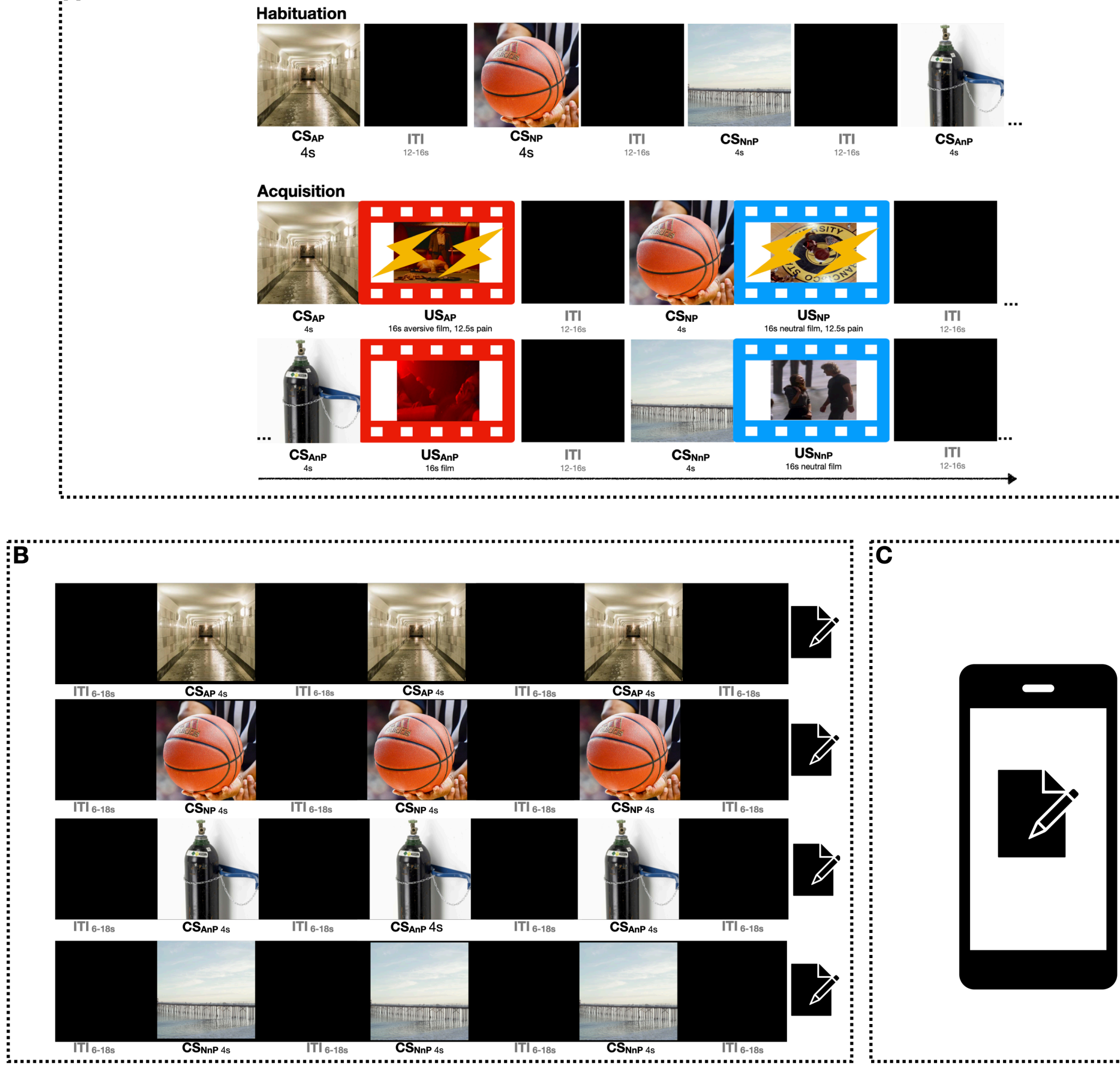

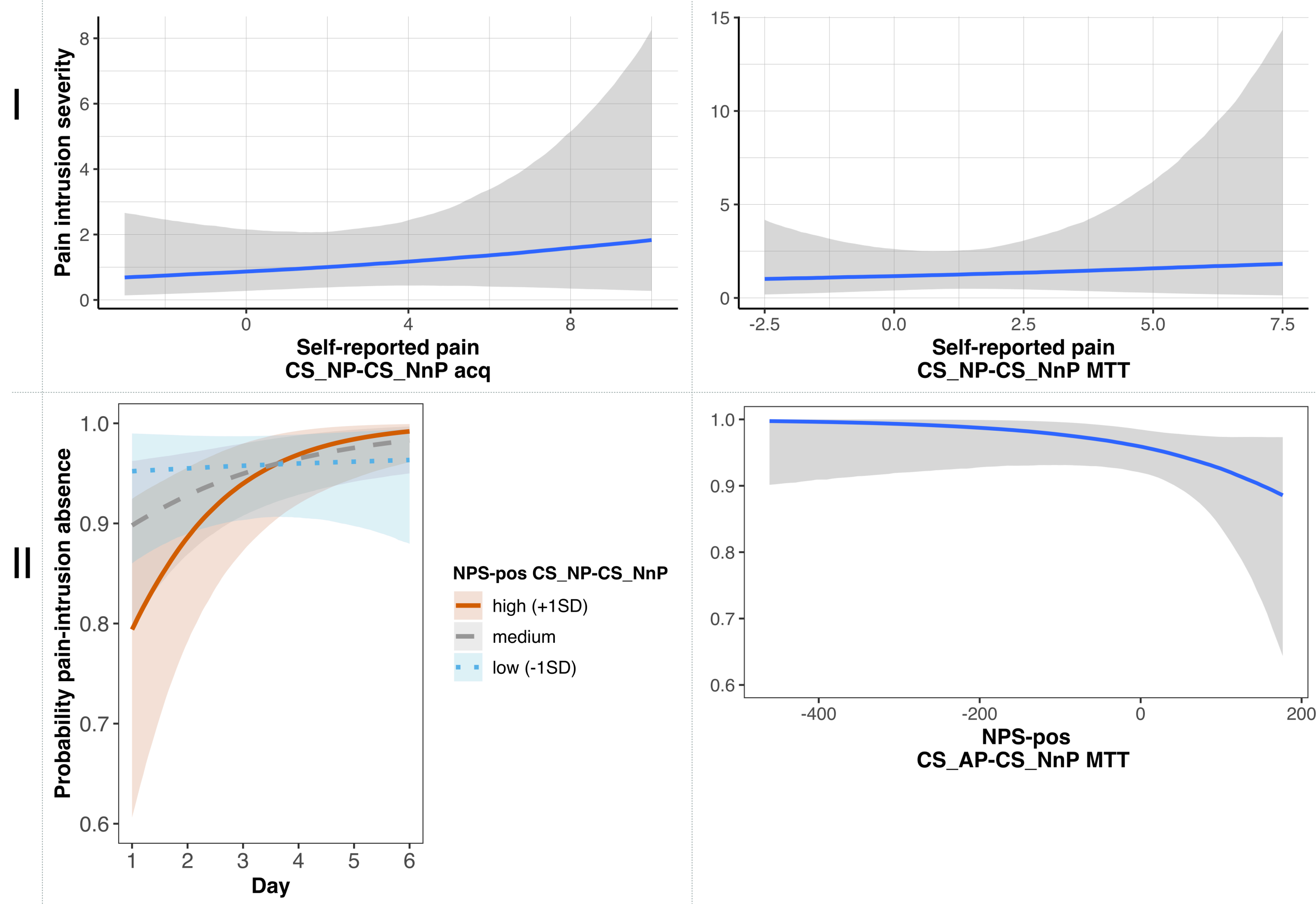

NPS-pos CS NP-CS NnP

- high (+1SD)

- medium

- " low (-1SD)

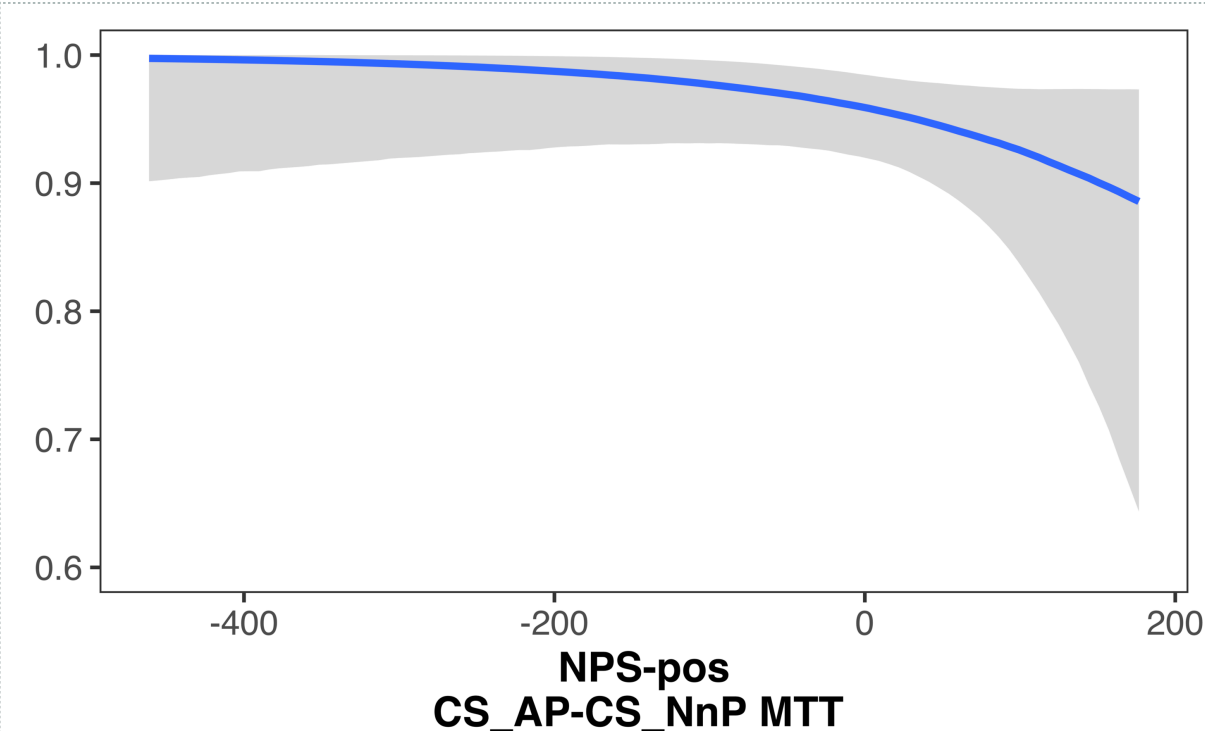




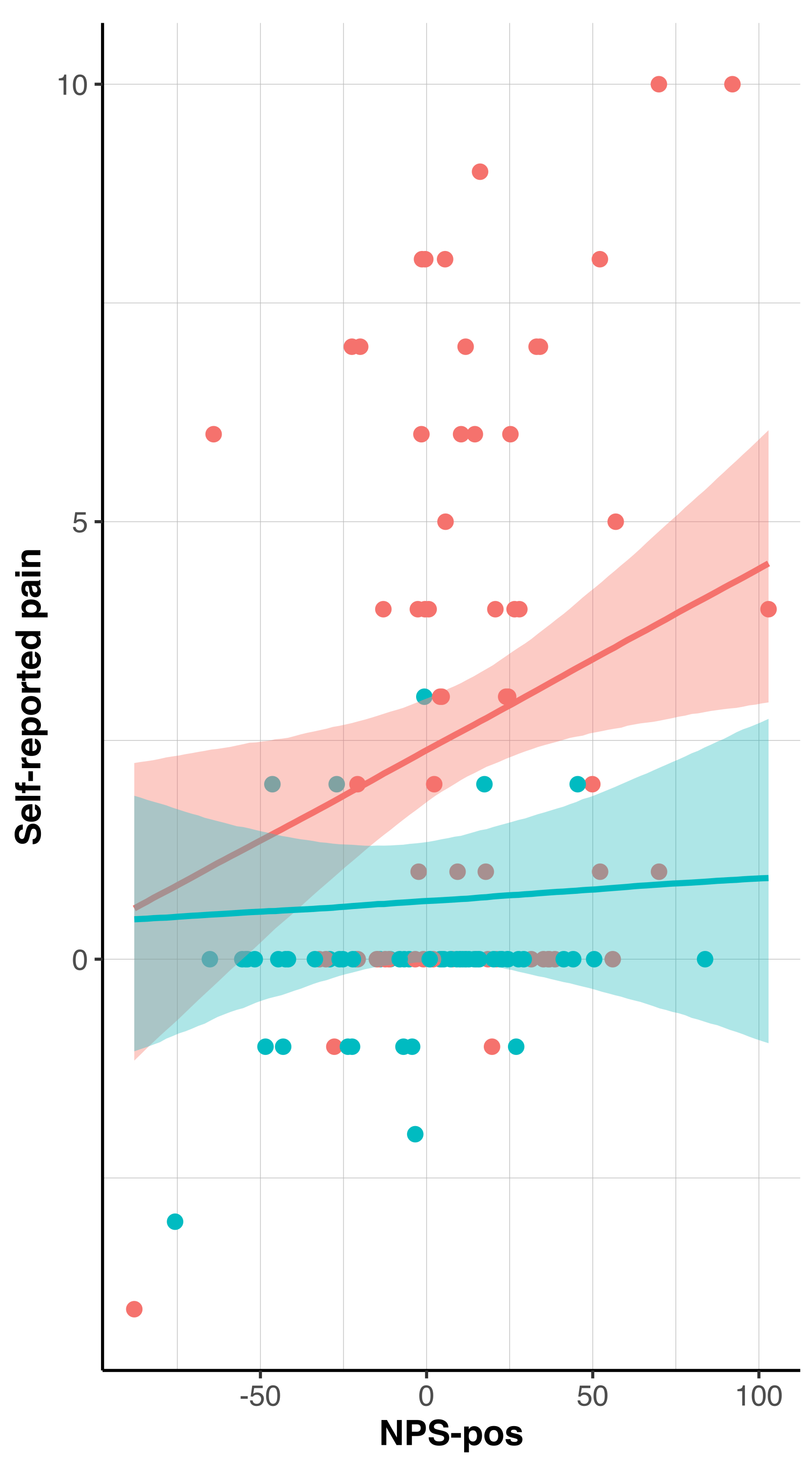

\section{Pain}

- pain

- nopain 
A

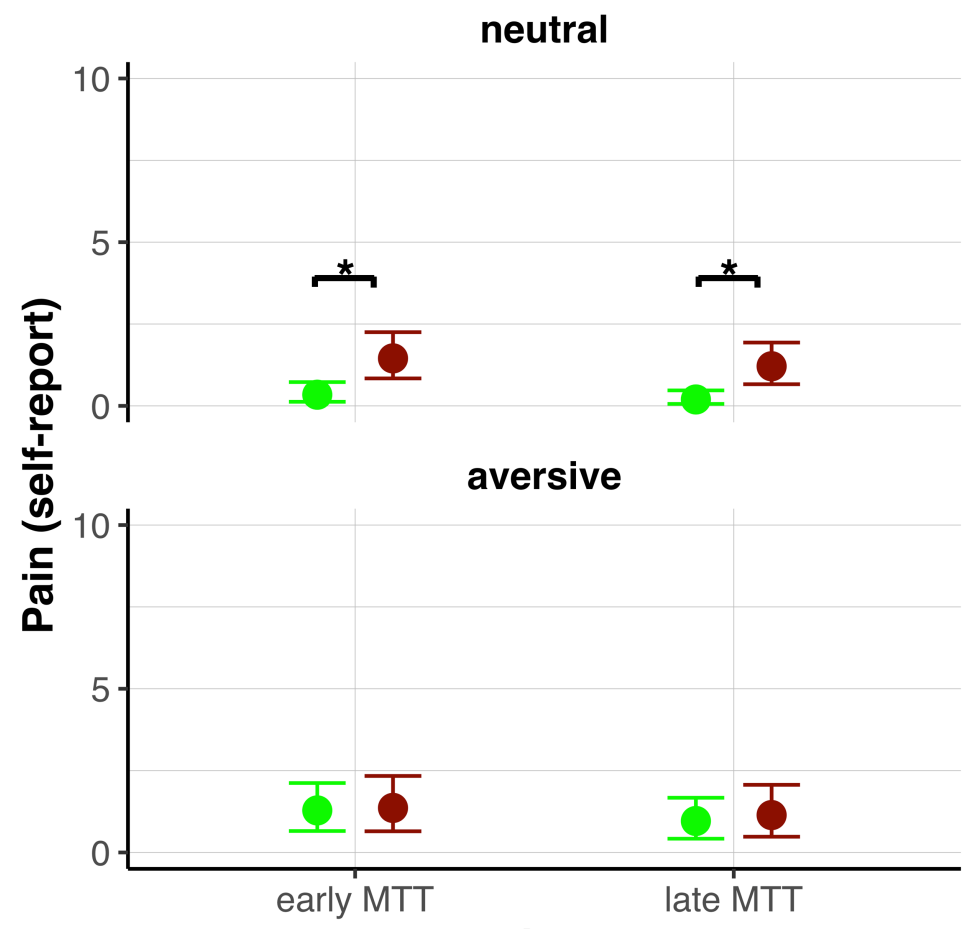

B

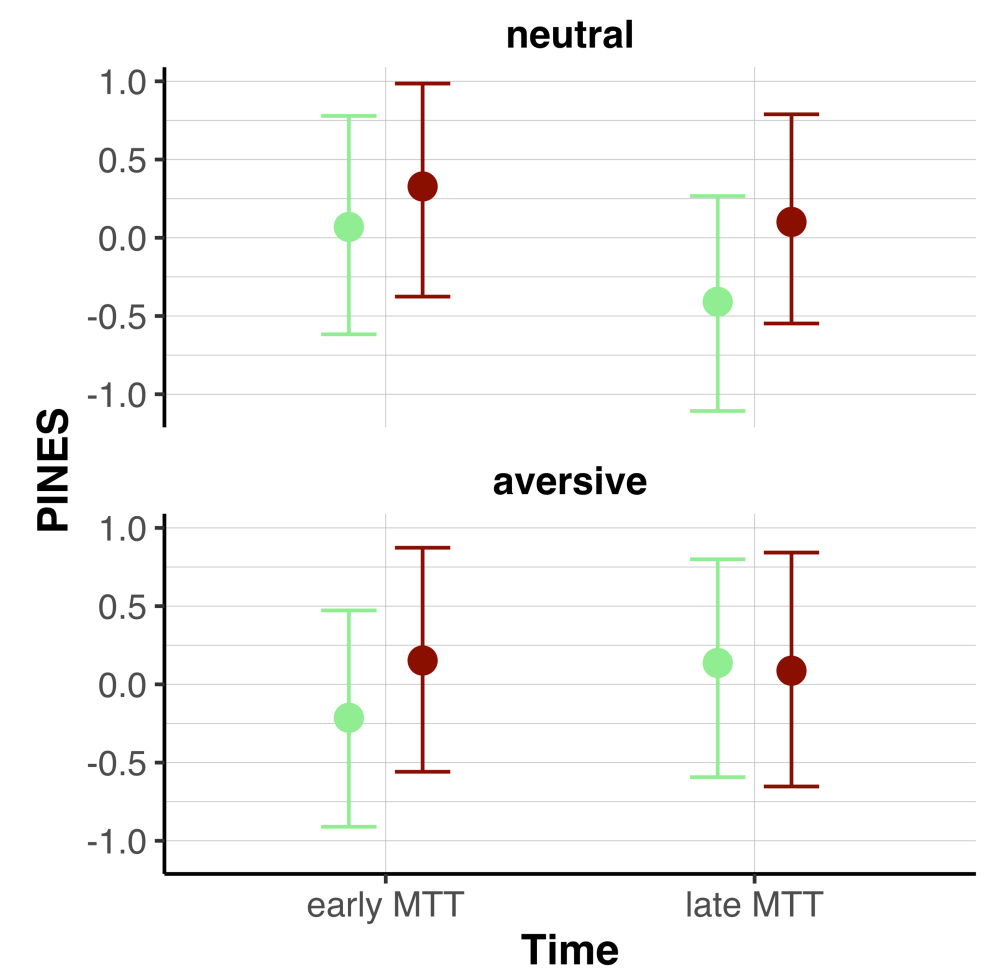

neutral

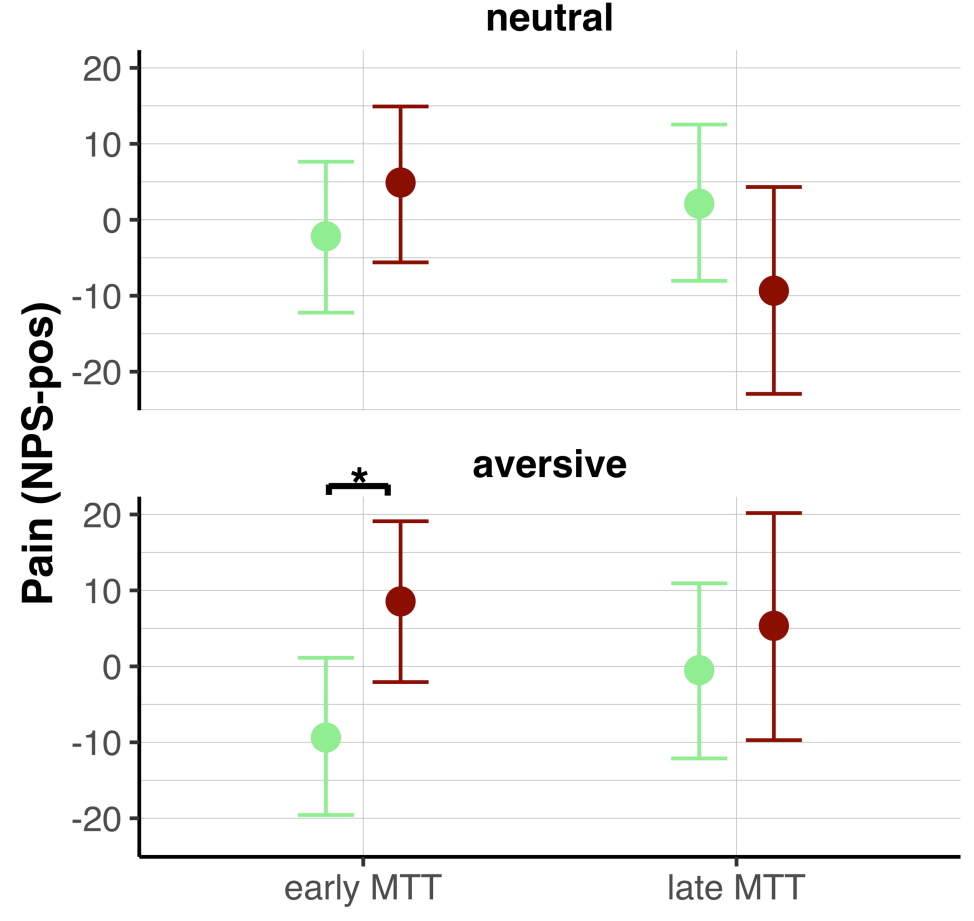

Pain

- no-pain

- pain

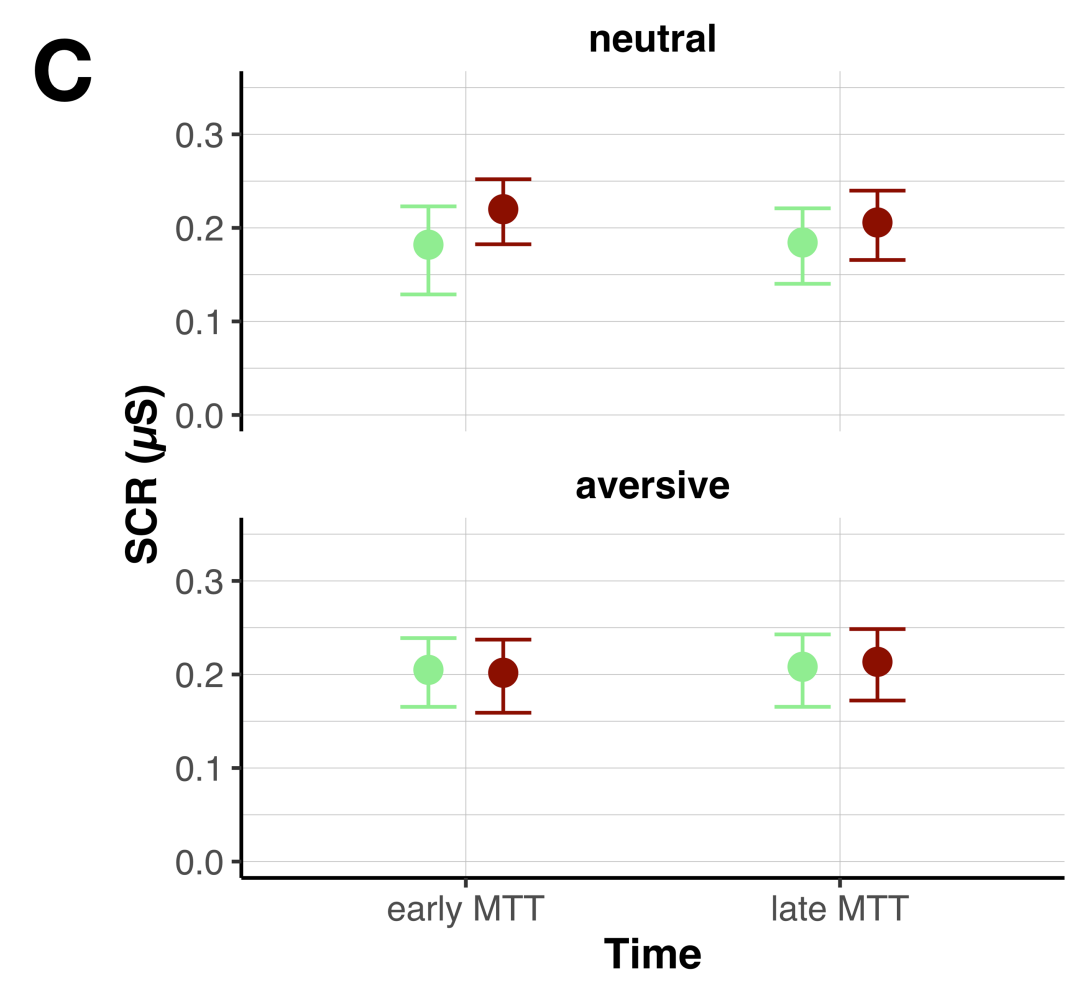



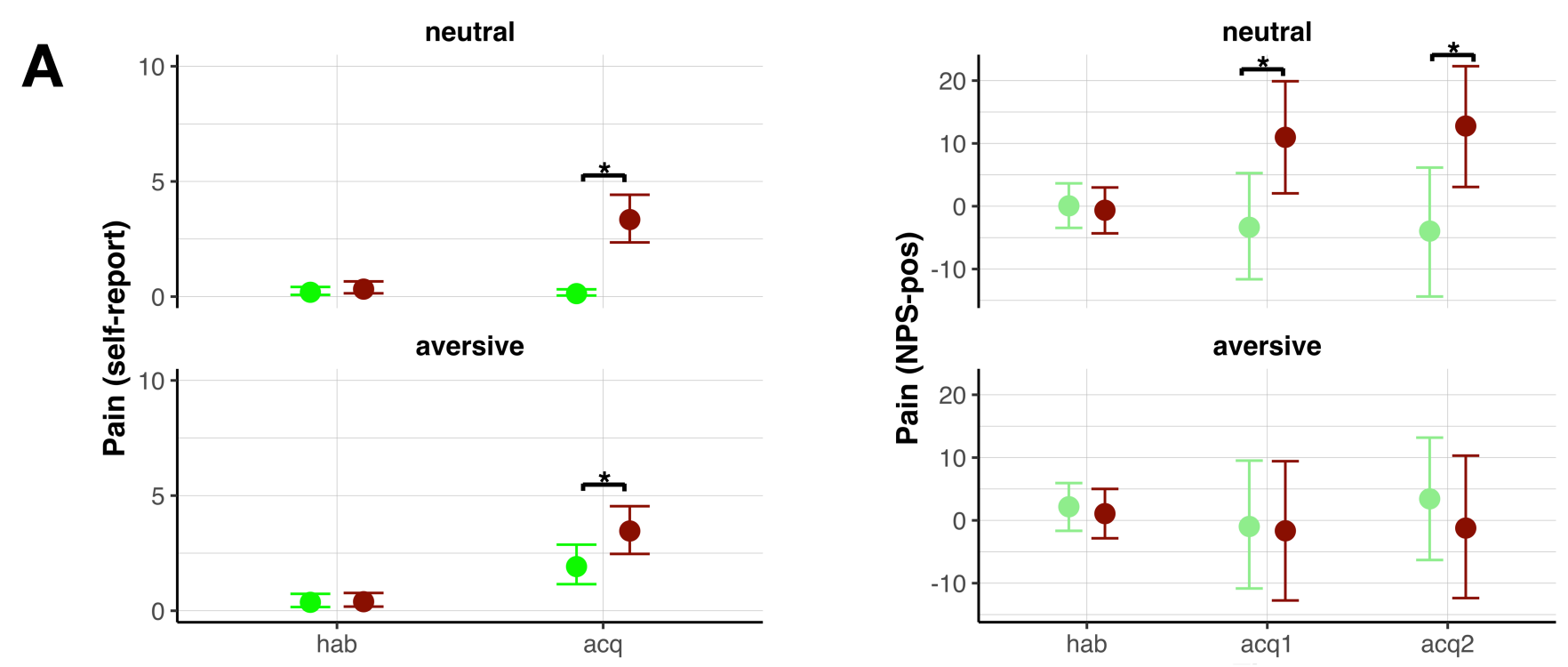

Pain

B
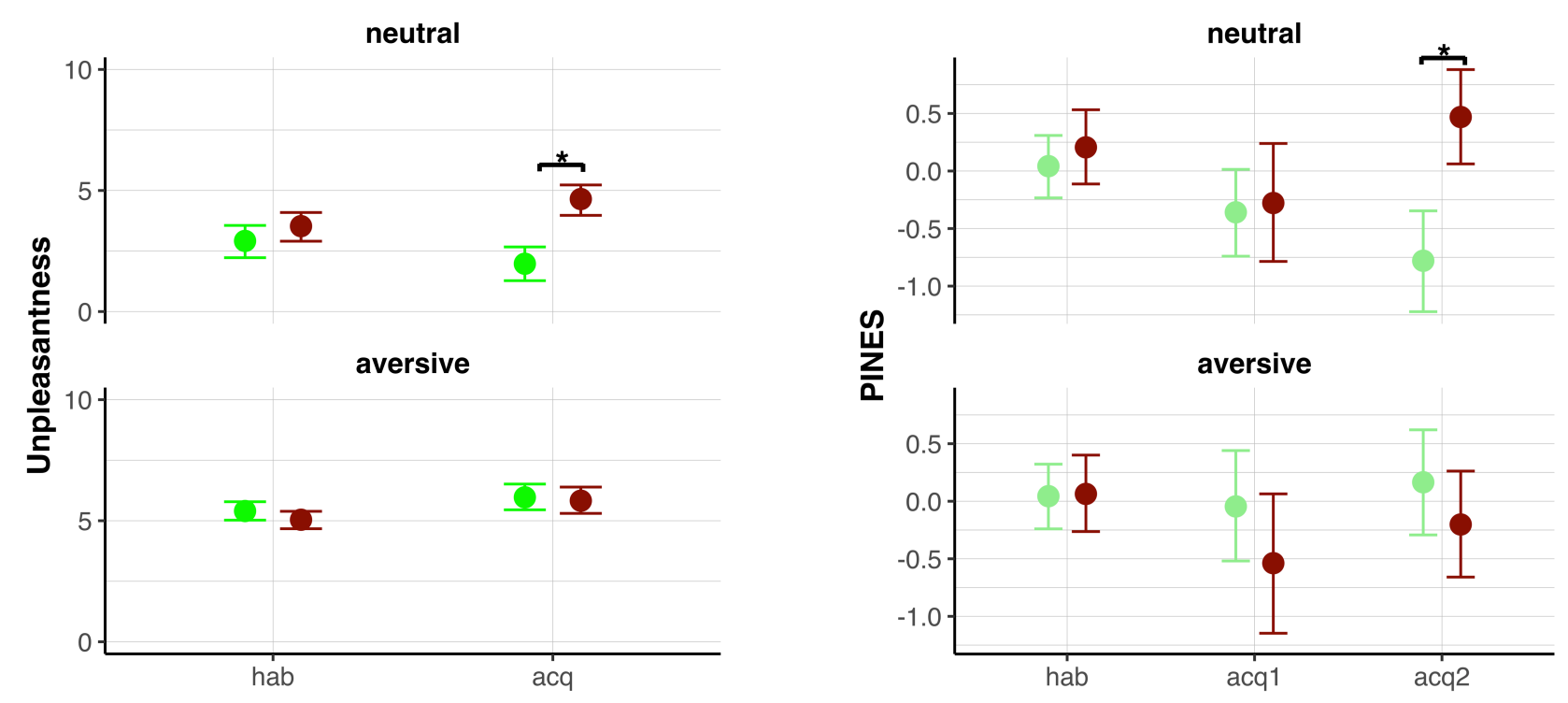

C
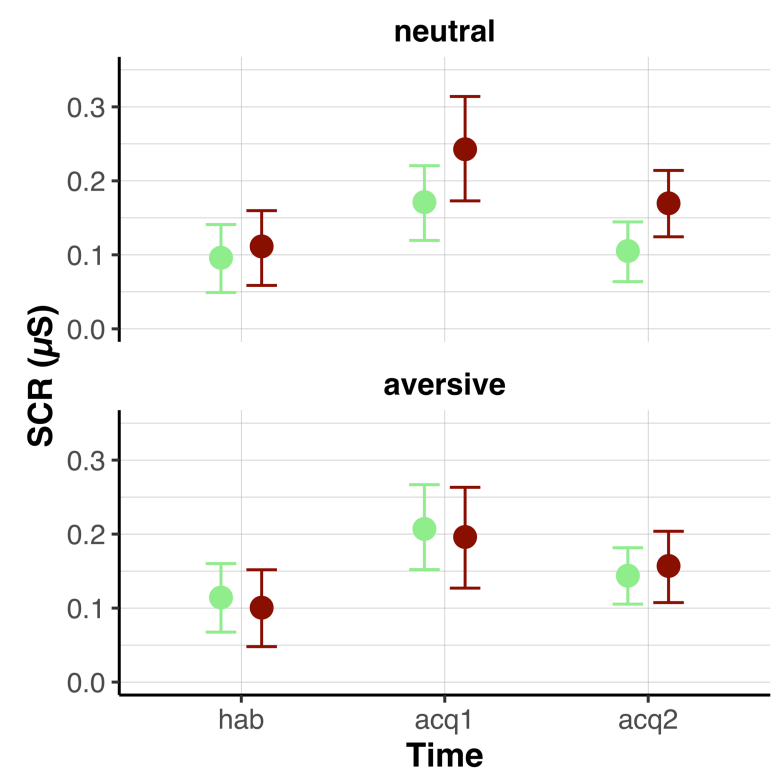

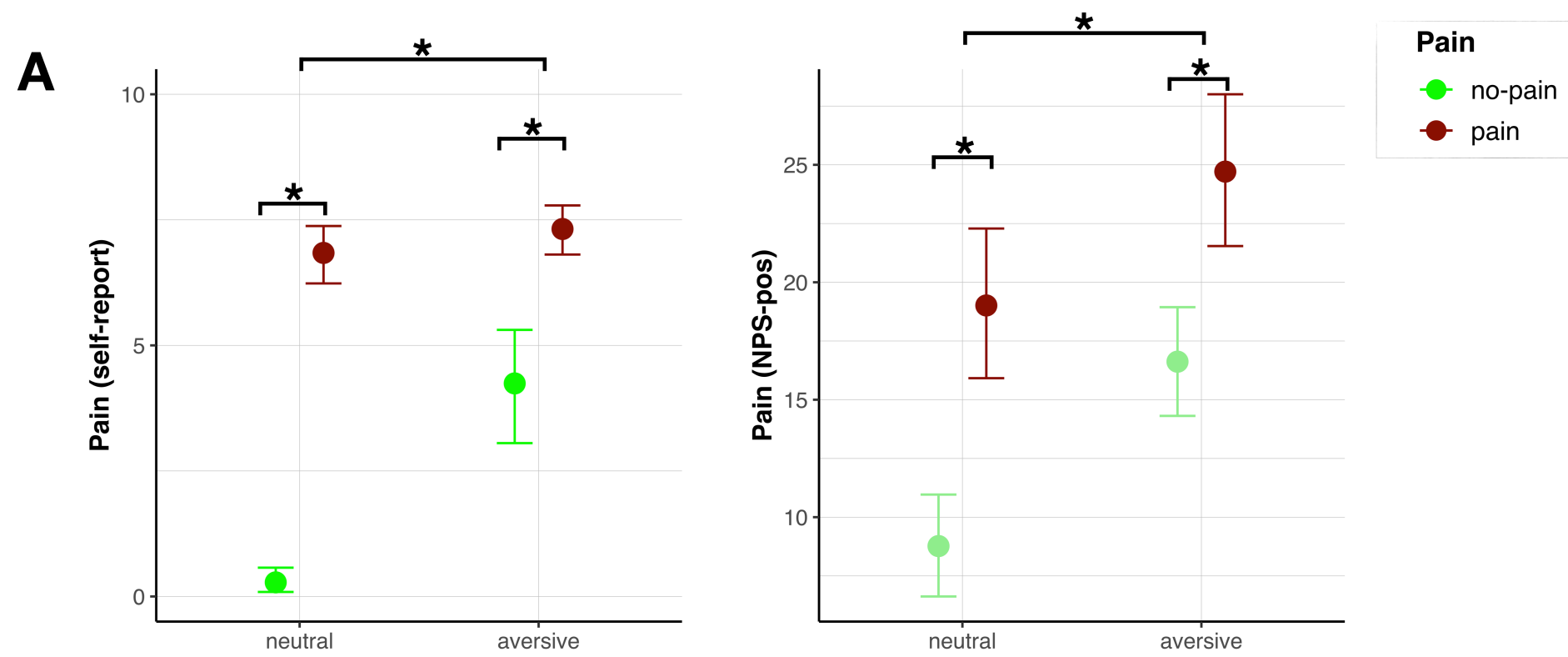

B

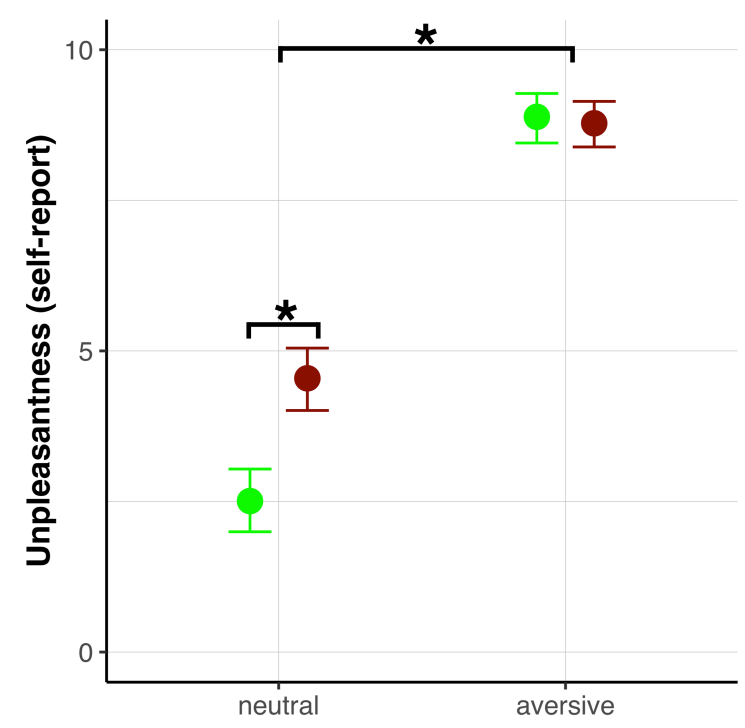

C

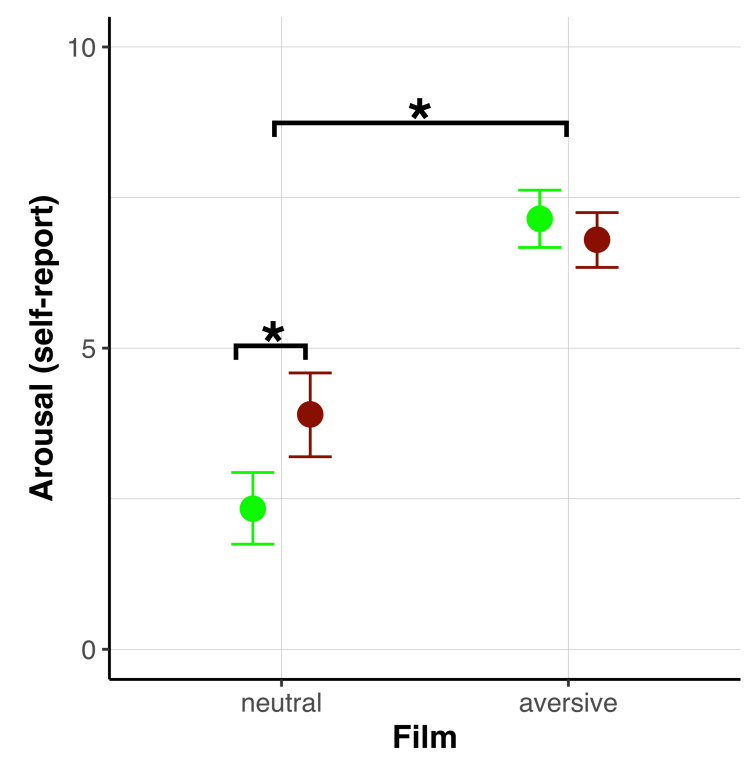

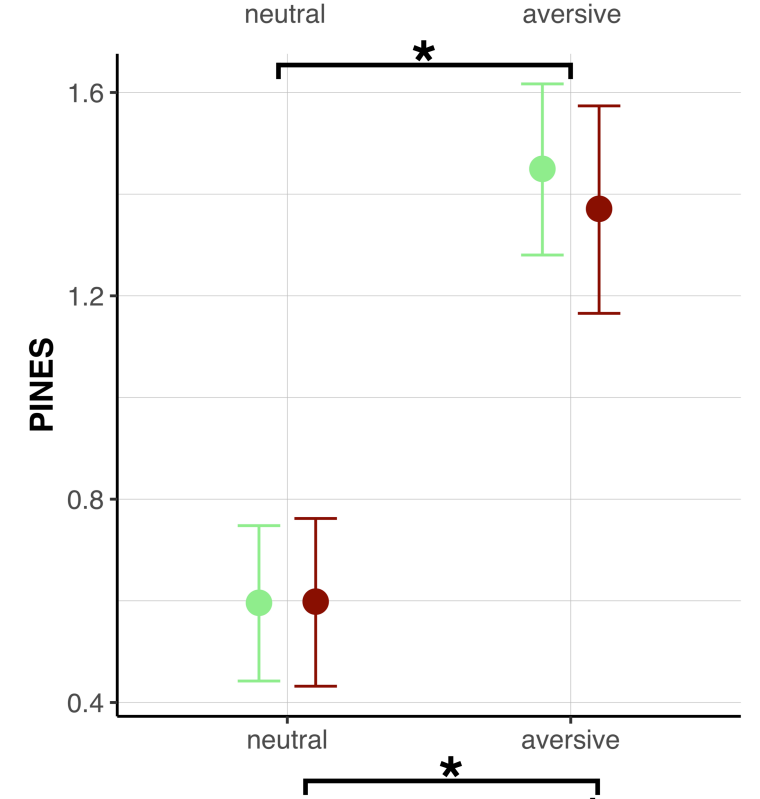

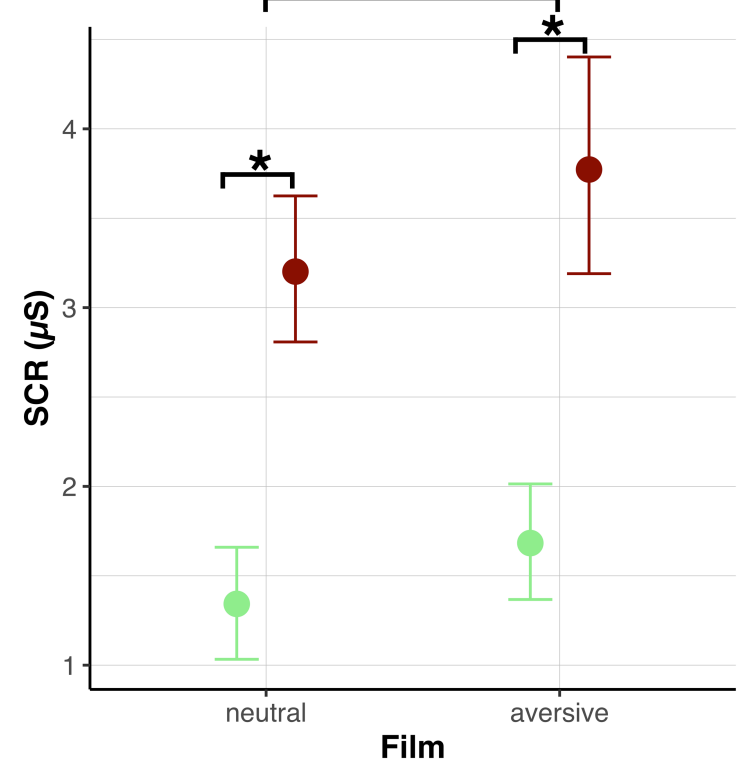




\section{Supplemental Digital Content}

\section{Supplement 1. Overview of statistical models and respective formulas}

Table S1. Overview of Bayesian Multilevel Models

A - Manipulation checks

Pain (I)

UR_self-rep_pain $\sim$ Pain $\times$ Film +

Pain (II)

$(1+$ Pain $\times$ Film $\mid$ Subject $)$, family $=$ cumulative

Pain (II)

UR_NPS-pos $\sim$ Pain $\times$ Film +

Neg. emo (I)

$(1+$ Pain $\times$ Film $\mid$ Subject $)$, family = gaussian

Neg. emo (II)

UR_valence $\sim$ Pain $\times$ Film +

$(1+$ Pain $\times$ Film $\mid$ Subject $)$, family = cumulative

UR_PINES $\sim$ Pain $\times$ Film +

$(1+$ Pain $\times$ Film $\mid$ Subject $)$, family = gaussian

Arousal (I)

UR_arousal $\sim$ Pain $\times$ Film +

Arousal (II)

$(1+$ Pain $\times$ Film $\mid$ Subject $)$, family = cumulative

UR_SCR $\sim$ Pain $\times$ Film +

$(1+$ Pain $\times$ Film $\mid$ Subject $)$, family $=$ skew_normal

B - Main analyses

CRs Pain (I)

acquisition

(TPC)

Pain (II)

CR_self-rep_pain $\sim$ Pain $\times$ Film $\times$ Time +

$(1+$ Pain $\times$ Film $\times$ Time $\mid$ Subject $)$, family $=$ cumulative

CR_NPS-pos $\sim$ Pain $\times$ Film $\times$ Time +

$(1+$ Pain $\times$ Film $\times$ Time $\mid$ Subject $)$, family $=$ gaussian

Neg. emo (I)

CR_valence $\sim$ Pain $\times$ Film $\times$ Time +

$(1+$ Pain $\times$ Film $\times$ Time $\mid$ Subject $)$, family $=$ cumulative

Neg. emo (II)

CR_PINES $\sim$ Pain $\times$ Film $\times$ Time +

$(1+$ Pain $\times$ Film $\times$ Time $\mid$ Subject $)$, family $=$ gaussian

SCRs

CR_SCRs $\sim$ Pain $\times$ Film $\times$ Time +

$(1+$ Pain $\times$ Film $\times$ Time $\mid$ Subject $)$, family $=$ skew_normal

$\begin{array}{lll}\text { CRs } & \text { Pain }(\mathrm{I}) & \text { CR_self-rep_pain } \sim \text { Pain } \times \text { Film } \times \text { Time }+ \\ \text { retention } & & (1+\text { Pain } \times \text { Film } \times \text { Time } \mid \text { Subject }), \text { family }\end{array}$

$(1+$ Pain $\times$ Film $\times$ Time $\mid$ Subject $)$, family $=$ cumulative

(MTT) Pain (II)

CR_NPS-pos $\sim$ Pain $\times$ Film $\times$ Time +

Neg. emo (I)

$(1+$ Pain $\times$ Film $\times$ Time $\mid$ Subject $)$, family $=$ gaussian

CR_PINES $\sim$ Pain $\times$ Film $\times$ Time +

$(1+$ Pain $\times$ Film $\times$ Time $\mid$ Subject $)$, family $=$ gaussian

SCRs

CR_SCRs $\sim$ Pain $\times$ Film $\times$ Time +

$(1+$ Pain $\times$ Film $\times$ Time $\mid$ Subject $)$, family $=$ skew_normal

C - Relationship between self-reported pain and NPS-pos CRs

Acquisition

CR_self-rep_pain $\sim$ CR_NPS-pos $\times$ Pain $\times$ Film +

$(1+$ Pain $\times$ Film $\mid$ Subject $)$, family $=$ skew_normal

MTT

CR_self-rep_pain $\sim$ CR_NPS-pos $\times$ Pain $\times$ Film + Time + $(1+$ Pain $\times$ Film $\mid$ Subject $)$, family $=$ cumulative 
D - Relationship between pain-CRs and pain-intrusions

\begin{tabular}{|c|c|}
\hline Acquisition & $\begin{array}{l}\text { Pain_ints } \sim \text { Day } \times \text { self-rep-pain-CRs_withinNeutral, } \\
\text { Hu } \sim \text { Day } \times \text { self-rep-pain-CRs_withinNeutral }+ \\
(1+\text { Day } \mid \text { Subject }), \text { family }=\text { hurdle_lognormal } \\
\text { Pain_ints } \sim \text { Day } \times \text { NPS-pos-pain-CRs_withinNeutral, } \\
\text { Hu } \sim \text { Day } \times \text { NPS-pos-pain-CRs_withinNeutral }+ \\
(1+\text { Day } \mid \text { Subject }), \text { family }=\text { hurdle_lognormal } \\
\text { Pain_ints } \sim \text { Day } \times \text { self-rep-pain-CRs_withinAversive, } \\
\text { Hu } \sim \text { Day } \times \text { self-rep-pain-CRs_withinAversive }+ \\
(1+\text { Day } \mid \text { Subject }), \text { family }=\text { hurdle_lognormal } \\
\text { Pain_ints } \sim \text { Day } \times \text { NPS-pos-pain-CRs_withinAversive, } \\
\text { Hu } \sim \text { Day } \times \text { NPS-pos-pain-CRs_withinAversive }+ \\
(1+\text { Day } \mid \text { Subject }), \text { family }=\text { hurdle_lognormal }\end{array}$ \\
\hline MTT & $\begin{array}{l}\text { Pain_ints } \sim \text { Day } \times \text { self-rep-pain-CRs_withinNeutral, } \\
\text { Hu } \sim \text { Day } \times \text { self-rep-pain-CRs_withinNeutral }+ \\
(1+\text { Day } \mid \text { Subject }), \text { family }=\text { hurdle_lognormal } \\
\text { Pain_ints } \sim \text { Day } \times \text { NPS-pos-pain-CRs_withinNeutral, } \\
\text { Hu } \sim \text { Day } \times \text { NPS-pos-pain-CRs_withinNeutral }+ \\
(1+\text { Day } \mid \text { Subject }), \text { family }=\text { hurdle_lognormal } \\
\text { Pain_ints } \sim \text { Day } \times \text { self-rep-pain-CRs_withinAversive, } \\
\text { Hu } \sim \text { Day } \times \text { self-rep-pain-CRs_withinAversive }+ \\
(1+\text { Day } \mid \text { Subject }), \text { family }=\text { hurdle_lognormal } \\
\text { Pain_ints } \sim \text { Day } \times \text { NPS-pos-pain-CRs_withinAversive, } \\
\text { Hu } \sim \text { Day } \times \text { NPS-pos-pain-CRs_withinAversive }+ \\
(1+\text { Day } \mid \text { Subject }), \text { family }=\text { hurdle lognormal }\end{array}$ \\
\hline
\end{tabular}

Note. In acquisition analyses, Time encoded the habituation and acquisition phases, yielding one level for self-report data (habituation/full acquisition) and three levels (habituation/early acquisition, late acquisition) in analyses estimating imaging data and SCRs. In MTT analyses, Time encoded early and late MTT blocks. For analyses D, we operationalized pain-CRs with difference scores between pain responses to the CSpain+neutral-film - CSnopain+neutral-film for pain-CRs within the neutral-film affective context ("withinNeutral"), and with difference scores between pain responses to the CSpain+aversive-film - CSnopain+neutral-film for pain-CRs within the aversive-film affective context ("withinAversive"). Abbreviations: UR = unconditioned response; $\mathrm{CR}=$ conditioned response; self-rep_pain = self-reported pain; NPS-pos $=$ neural pain signature with positive weights only; SCRs=skin conductance responses; TPC = Trauma-Pain-Conditioning procedure; MTT = Memory-Triggering-Task. 


\section{Supplement 2. Results with NPS-responses excluding occipital cortex [manually edited} NPS mask in SPM]

\section{S2a. Acquisition}

Results were comparable to NPS responses derived from the NPS-pos mask. Specifically, NPS [without occipital regions] responses to CSs revealed a significant Pain $\times$ Time_acq1 interaction. The interaction between Pain $\times$ Time_acq2 was in the same direction but associated with somewhat more uncertainty (see Table S2A for regression coefficients and 95\%-CIs). As displayed in Fig. S2 and corroborated by post-hoc analyses on NPS responses to CSs during each study timepoint (habituation/acq1+acq2), these interactions suggested that participants showed greater NPS responses $\mathrm{CS}_{\text {pain }}$ than $\mathrm{CS}_{\text {nopain }}(b=0.32,95 \%-\mathrm{CI}=[0.10$, 0.61]): The non-significant Pain $\times$ Acquisition-time (acq1+acq2) interaction suggested that the main-effect of Pain held across both acq1 and acq2 $(b=-0.06,95 \%-\mathrm{CI}=[-0.52,0.39])$. During habituation, participants did not show significantly greater NPS responses to $\mathrm{CS}_{\text {pain }}$ than $\mathrm{CS}_{\text {nopain }}(b=-0.06,95 \%-\mathrm{CI}=[-0.21,0.09])$. Post-hoc analyses within each study phase (hab/acq1+acq2) and film condition further suggested that within the neutral-film condition participants showed significantly greater NPS responses to $\mathrm{CS}_{\text {pain }}$ than $\mathrm{CS}_{\text {nopain }}$ during acq1 (b $=0.36,95 \%-\mathrm{CI}=[0.02,0.69])$. During acq 2 , this effect was associated with somewhat more uncertainty but nevertheless pointed in the same direction $(b=0.29,95 \%-\mathrm{CI}=[-0.06,0.64])$. Within the aversive-film condition, participants did not show significantly greater NPS responses to $\mathrm{CS}_{\text {pain }}$ than $\mathrm{CS}_{\text {nopain }}$ during acq1 $(b=0.13,95 \%-\mathrm{CI}=[-0.20,0.45])$ or acq2 $(b=-$ $0.16,95 \%-\mathrm{CI}=[-0.31,0.36])$. 


\section{S2b. MTT}

As can be seen in Table S2B and Fig. S2B, analyses revealed that NPS responses were stronger during the $\mathrm{CS}_{\text {pain }}$ than $\mathrm{CS}_{\text {nopain }}$ conditions. There were no significant interaction between Pain, Film, and Time.

Table S2. Effects of Pain, Film-Valence, and Time on NPS-responses calculated with a mask excluding voxels in the occipital cortex during (A) Acquisition and (B) MTT.

\begin{tabular}{|c|c|c|c|c|}
\hline & $b$ & $95 \%-C I$ & $R^{2}$ & $95 \%-C I$ \\
\hline (A) Acquisition & & & & \\
\hline Pain & -0.05 & {$[-0.22,0.11]$} & 0.19 & {$[0.12,0.25]$} \\
\hline Film & 0.07 & {$[-0.10,0.23]$} & & \\
\hline Time_acq1 & 0.02 & {$[-0.28,0.31]$} & & \\
\hline Time_acq2 & 0.11 & {$[-0.20,0.44]$} & & \\
\hline Pain $\times$ Film & 0.06 & {$[-0.17,0.29]$} & & \\
\hline Pain $\times$ Time_acq1 & 0.46 & {$[0.07,0.87]$} & & \\
\hline Pain $\times$ Time_acq2 & 0.38 & {$[-0.04,0.78]$} & & \\
\hline Film $\times$ Time_acq1 & -0.09 & {$[-0.52,0.34]$} & & \\
\hline Film $\times$ Time_acq2 & -0.06 & {$[-0.47,0.34]$} & & \\
\hline Pain $\times$ Film $\times$ Time_acq 1 & -0.30 & {$[-0.89,0.28]$} & & \\
\hline Pain $\times$ Film $\times$ Time_acq2 & -0.35 & {$[-0.92,0.24]$} & & \\
\hline (B) $\quad M T T$ & & & 0.28 & {$[0.18,0.38]$} \\
\hline Pain & 0.35 & {$[0.05,0.65]$} & & \\
\hline Film & 0.06 & {$[-0.24,0.37]$} & & \\
\hline Time_mtt2 & 0.10 & {$[-0.20,0.41]$} & & \\
\hline Pain $\times$ Film & -0.10 & {$[-0.53,0.33]$} & & \\
\hline Pain $\times$ Time_mtt2 & -0.17 & {$[-0.63,0.29]$} & & \\
\hline Film $\times$ Time_mtt2 & -0.08 & {$[-0.51,0.35]$} & & \\
\hline Pain $\times$ Film $\times$ Time_mtt2 & -0.25 & {$[-0.94,0.45]$} & & \\
\hline
\end{tabular}

Note. Coefficients are considered significantly different from zero if the corresponding $95 \%$ CI does not contain zero and are highlighted in bold. For improved readability, we do not display intercepts. Abbreviations: acq $=$ acquisition; NPS-pos $=$ neural pain signature with positive weights only; SCRs = skin conductance responses. 

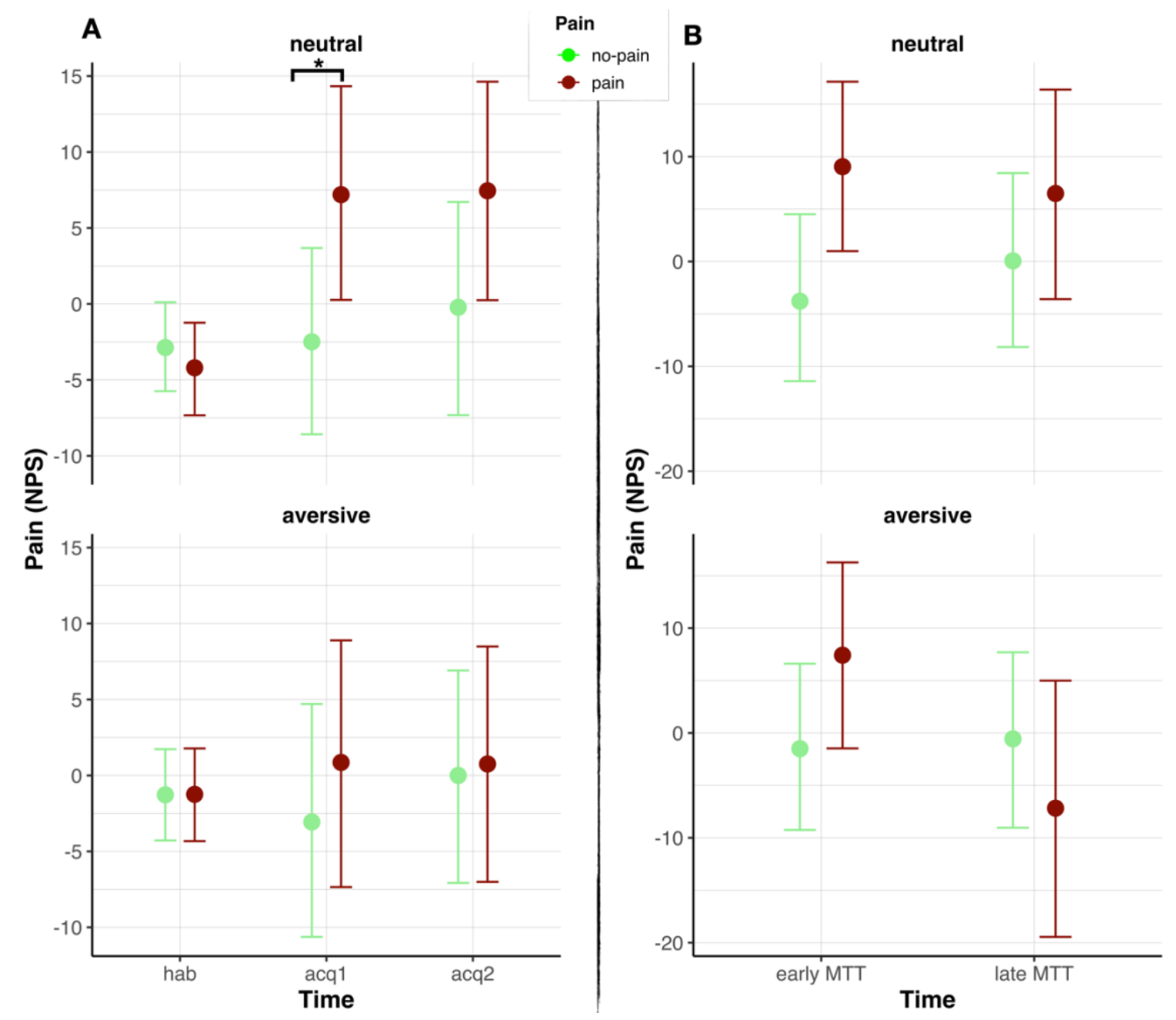

Figure S2. Conditioned NPS responses (CRs) to each CS condition during Acquisition (left panel A) and Memory Triggering Task (MTT, right panel B). Asterisks mark under which study phase (hab/acq1/acq2) and affective film-condition (aversive/neutral) the $\mathrm{CS}_{\text {pain }}$ vs. CSnopain comparisons were significantly different from zero as determined by post-hoc analyses. Abbreviations: hab = habituation; acq $=$ acquisition with trials averaged over both acquisition halves; acq 1 = first acquisition halve; acq $2=$ second acquisition halve; MTT = Memory Triggering Task. 


\section{Supplement 3. Whole-brain analyses on CS-activations.}

\section{Method:}

For all analyses, the threshold was set to $\mathrm{p}<.05$ corrected for multiple comparisons (based on the false discovery rate (FDR), whole brain level), and a cluster size of $\mathrm{k} \geq 5$.

\section{Results acquisition:}

Main effect of Film during acquisition (F-test; FDR-corrected $p<0.05 ; \mathrm{k} \geq 5$ ).

No effects.

Main effect of Pain during acquisition (F-test; FDR-corrected $p<0.05 ; \mathrm{k}=5$ ).

No effects.

Table S3a. Interaction Pain $\times$ Film during acquisition (F-test; FDR-corrected $p<0.05 ; \mathrm{k} \geq 5$ ).

\begin{tabular}{lccc} 
Region & $\begin{array}{c}\text { Cluster Size, } \\
\text { Voxels }\end{array}$ & z Score & MNI coordinates, (x, $\mathbf{~}$ \\
\hline R Middle Temporal/Occipital Gyrus & 2667 & Inf & , z) \\
& & 6.70 & $42,-66,4$ \\
L Occipital Gyrus & 1175 & 6.49 & $38,-82,6$ \\
L Fusiform Gyrus & & Inf & $-42,-72,8$ \\
& & 5.44 & $-34,-80,-16$ \\
L Superior Temporal Gyrus & 695 & 5.34 & $-28,-72,-6$ \\
L Inferior Parietal Lobe & & 7.78 & $-52,-20,4$ \\
L Superior Temporal Gyrus & & 6.15 & $-44,-38,26$ \\
L Rolandic Operculum & & 5.53 & $-42,-32,10$ \\
R Superior Temporal Gyrus & 825 & 5.20 & $-38,-36,18$ \\
& & 6.47 & $62,-12,4$ \\
& & 6.45 & $64,-32,14$ \\
R Rolandic Operculum & & 6.02 & $52,-18,6$ \\
L Cerebellum & 17 & 4.89 & $54,-18,10$ \\
L Lingual Gyrus & 17 & 5.41 & $-10,-72,-40$ \\
R Precentral Gyrus & 101 & 5.38 & $-4,-60,0$ \\
& & 5.20 & $42,-2,56$ \\
L Occipital Gyrus & 54 & 5.19 & $44,-2,46$ \\
& & 5.13 & $-16,-86,30$ \\
& & 4.68 & $-22,-88,22$
\end{tabular}


L Anterior Insula
L Occipital Gyrus

L Brainstem

R Precentral Gyrus

$\mathrm{R}$ Anterior Insula

$\mathrm{R}$ Cerebellum

R Lingual Gyrus

L Lingual Gyrus

L Superior Temporal Gyrus

L Calcarine sulcus

$\mathrm{R}$ Inferior Frontal Cortex

R Cerebellum

L Lingual Gyrus

R Supplementary Motor Area
10

16
5.09

4.98

4.90

4.80

4.51

4.78

4.78

4.75

4.72

4.70

4.69

4.68

4.61

4.59

4.58

4.55
$-30,26,6$

$-10,-82,42$

$-18,-74,32$

$-12,-24,-10$

$-6,-30,-10$

$44,4,32$

$34,28,4$

4, $-70,-30$

$12,-58,2$

$-4,-82,2$

$-60,-44,12$

$-10,-74,12$

$42,16,22$

$8,-58,-38$

$-8,-74,-8$

$8,12,54$

Table $S 3 b$. Pain > NoPain within neutral-film condition during acquisition (t-test; FDR-

corrected $p<0.05 ; \mathrm{k} \geq 5$ ).

\section{Region \\ L Occipital Gyrus \\ L Fusiform Gyrus \\ L Superior Temopral Gyrus}

R Middle Temporal/Occipital Gyrus

R Superior Temoporal Gyrus

L Anterior Insula

R Anterior Insula

L Cerebellum

R Cerebellum

R Precentral Gyrus

L Precentral Gyrus

L Inferior Parietal Lobe

$\begin{array}{ccc}\begin{array}{c}\text { Cluster Size, } \\ \text { Voxels }\end{array} & \text { z Score } & \text { MNI coordinates, }(\mathbf{x}, \mathbf{y} \\ \mathbf{, ~ z})\end{array}$




\begin{tabular}{lccc} 
R Precuneus & 18 & 4.90 & $8,-46,52$ \\
R Cerebellum & 31 & 4.90 & $10,-58-38$ \\
R Occipital Gyrus & 19 & 4.89 & $22,-72,40$ \\
R Supplementary Motor Area & 54 & 4.87 & $8,16,54$ \\
& & 4.83 & $4,30,50$ \\
L Supramarginal Gyrus & 9 & 4.83 & $-60,-24,32$ \\
R Cingulate Gyrus & 14 & 4.83 & $12,20,36$ \\
R Superior Frontal Gyrus & 10 & 4.80 & $12,-2,72$ \\
L Superior Temporal Gyrus & 12 & 4.71 & $-60,-44,12$ \\
L Cerebellum & 12 & 4.66 & $-14,-74-22$ \\
L Rolandic Operculum & 13 & 4.64 & $-48,6,4$ \\
L Occipital Gyrus & 15 & 4.61 & $-22,-62-10$ \\
R Brainstem & 5 & 4.60 & $6,-32-24$ \\
L Lingual Gyrus & 8 & 4.58 & $-4,-82,2$ \\
R Cerebellum & 19 & 4.54 & $26,-70-30$ \\
R Cerebellum & 7 & 4.49 & $24,-60-28$ \\
R Cerebellum & 7 & 4.49 & $34,-58-24$ \\
R Superior Temoporal Gyrus & 6 & 4.47 & $52,2,-6$ \\
L Calcarine & 5 & 4.46 & $-8,-74,14$ \\
R Inferior Frontal Gyrus & 7 & 4.44 & $56,14,6$ \\
\hline
\end{tabular}

Pain $>$ NoPain within aversive-film condition revealed no sig. effects at FDR-corrected $p<$ $0.05 ; \mathrm{k} \geq 5$.

\section{Results MTT:}

Early MTT: No effect of Film x Pain interaction, main effect of Film and main effect of Pain (F-tests; FDR-corrected $p<0.05 ; \mathrm{k} \geq 5$ ).

Late MTT: No effect of Film x Pain interaction, main effect of Film and main effect of Pain (F-tests; FDR-corrected $p<0.05 ; \mathrm{k} \geq 5$ ). 